ARTICLE

\title{
Direct 1,3-butadiene biosynthesis in Escherichia coli via a tailored ferulic acid decarboxylase mutant
}

\author{
Yutaro Mori ${ }^{1}$, Shuhei Noda ${ }^{1}$, Tomokazu Shirai (iD) ${ }^{1 凶} \&$ Akihiko Kondo ${ }^{1,2,3}$
}

The C4 unsaturated compound 1,3-butadiene is an important monomer in synthetic rubber and engineering plastic production. However, microorganisms cannot directly produce 1,3butadiene when glucose is used as a renewable carbon source via biological processes. In this study, we construct an artificial metabolic pathway for 1,3-butadiene production from glucose in Escherichia coli by combining the cis,cis-muconic acid (ccMA)-producing pathway together with tailored ferulic acid decarboxylase mutations. The rational design of the substratebinding site of the enzyme by computational simulations improves ccMA decarboxylation and thus 1,3-butadiene production. We find that changing dissolved oxygen (DO) levels and controlling the $\mathrm{pH}$ are important factors for 1,3-butadiene production. Using DO-stat fedbatch fermentation, we produce $2.13 \pm 0.17 \mathrm{~g} \mathrm{~L}^{-1} 1$,3-butadiene. The results indicate that we can produce unnatural/nonbiological compounds from glucose as a renewable carbon source via a rational enzyme design strategy.

\footnotetext{
${ }^{1}$ Center for Sustainable Resource Science, RIKEN, Yokohama, Japan. ${ }^{2}$ Department of Chemical Science and Engineering, Graduate School of Engineering, Kobe University, Kobe, Japan. ${ }^{3}$ Graduate School of Science, Technology and Innovation, Kobe University, Kobe, Japan. ${ }^{凶}$ email: tomokazu.shirai@riken.jp
} 
$\mathrm{n}$ efforts to meet the United Nations sustainable development goals to solve environmental problems, biorefinery processes in which renewable nonfood feedstocks are used as raw materials are being used instead of petrochemical refinery processes to prevent fossil fuel depletion. With recent advances in genomeediting technology, synthetic biology has achieved the ability to produce desired compounds via engineered microorganisms ${ }^{1,2}$. Many researchers have investigated the microbial production of valuable chemicals that are currently produced from petroleum, such as fuels, bulk chemicals, amino acids, and pharmaceuticals ${ }^{3-8}$. Many studies have aimed to improve target compound yields by enzyme screening and enzyme engineering 9 . Furthermore, in industrial biotechnology, fermentation and distillation/recovery processes are important factors, as is the development of microorganisms that produce target compounds. Although the development of synthetic biology has led to the biosynthesis of many compounds ${ }^{10,11}$, biological production of novel unnatural/nonbiological compounds remains challenging.

1,3-Butadiene is a C4-conjugated diene with the simplest structure and is one of the most valuable industrial raw materials used for producing synthetic rubber (e.g., styrene-butadiene rubber and polybutadiene rubber) and engineering plastic (e.g., acrylonitrile-butadiene-styrene resin) ${ }^{12}$. The global market of 1,3-butadiene was $\$ 19$ billion in 2019 , and its global annual demand is $>12$ million metric tons. Nearly all 1,3-butadiene is typically synthesized from a $\mathrm{C} 4$ fraction that is a byproduct of naphtha cracking during ethylene production ${ }^{13}$. However, given that inexpensive ethylene derived from shale gas is currently produced, the supply of 1,3-butadiene has declined owing to the reduced production of ethylene derived from naphtha, and the gap between the supply and demand of 1,3-butadiene is predicted to increase ${ }^{14}$. To solve this problem and generate a sustainable society, there is a demand for switching from petroleum-based to biobased 1,3-butadiene.

In recent years, research on biobased 1,3-butadiene production has increased ${ }^{15}$. However, these are chemically synthesized products from biobased ethanol, 1- or 2-butanol, 1,3- or 2,3-butanediol, or tetrahydrofuran ${ }^{16-18}$. The existence of three different pathways have been suggested for 1,3-butadiene production from glucose via crotonyl-CoA, erythrose-4-phosphate, and malonyl$\mathrm{CoA}^{19}$, but the direct 1,3-butadiene biosynthesis from glucose as a renewable carbon source has not been achieved.

Muconic acid is an unsaturated six-carbon carboxylic acid with a conjugated diene and exists as three different isomers: cis,cismuconic acid (ccMA), cis,trans-muconic acid (ctMA), and trans, trans-muconic acid $(t t \mathrm{MA})^{20}$. ccMA is a natural metabolite involved in the decomposition pathway of aromatic compounds, such as benzoic acid derivatives, and can be produced via multiple pathways with different intermediate compounds ${ }^{4,21-23}$. Here, it was assumed that 1,3-butadiene can be produced by two decarboxylation reactions of $c c \mathrm{MA}$ (Fig. 1).

Ferulic acid decarboxylase (FDC), a member of the UbiD family enzymes, mediates the decarboxylation of phenylacrylic acid derivatives and converts them to terminal alkenes ${ }^{24}$. Cofactor prenylated flavin mononucleotide (prFMN) was recently discovered, and it was revealed that FDC-binding prFMN catalyzes the decarboxylation reaction ${ }^{25}$. prFMN is biosynthesized from FMN and dimethylallyl monophosphate by prenyltransferases encoded by the $u b i X$ and pad1 genes. It was revealed that UbiD family enzymes encoded by the $u b i D$ and $f d c 1$ genes may be associated with prFMN as a cofactor ${ }^{26}$. prFMNbinding FDC can recognize not only aromatic compounds but also $\alpha, \beta$-unsaturated carboxylic acids to produce terminal alkenes $^{27-36}$. Therefore, this FDC was selected as a template enzyme for producing butadiene from the $\alpha, \beta$-unsaturated dicarboxylic acid $c c \mathrm{MA}$.
Directed enzyme evolution with random mutagenesis is a powerful method, but it can be time consuming $37-40$. Thus, to narrow the mutant library, rational enzyme design based on in silico calculations is attractive. Changing the substrate specificity and improving enzymatic activities against unnatural substrates are reported with this strategy ${ }^{41-43}$.

In this study, we perform a rational enzyme design based on the affinity between FDC and $c c \mathrm{MA}$ and tailored FDC to synthesize 1,3-butadiene. We select FDCs derived from Aspergillus niger and Saccharomyces cerevisiae (AnFDC and ScFDC, respectively). The $c c \mathrm{MA}$ decarboxylation reaction is achieved via aminoacid substitution to capture $c c \mathrm{MA}$ at the FDC substrate-binding site while conserving the amino-acid residues involved in the decarboxylation reaction. Next, by combining the $c c \mathrm{MA}$ producing pathway with tailored mutant FDCs in Escherichia coli, we construct an artificial metabolic pathway for 1,3-butadiene production from glucose. By balancing the aerobic phase for $c c$ MA production and the microaerobic phase for 1,3-butadiene production, we succeed in the direct production of 1,3-butadiene from glucose.

\section{Results}

Designing AnFDC mutants for 1,3-butadiene biosynthesis from ccMA. AnFDC was selected as a template decarboxylase for 1,3-butadiene production. Decarboxylation by AnFDC requires prFMN, Q282, and R173, and the substrate specificity of $A n \mathrm{FDC}$ is determined by the other surrounding residues in the substrate-binding site. The model of a-methyl cinnamic acid-bound $A n$ FDC showed that R173 interacts with the carboxylate $_{\text {react. }}$ group of $\alpha$-methyl cinnamic acid (Fig. $\left.2 \mathrm{a}\right)$. In the substrate-binding site of FDC, hydrophobic amino-acid residues (L185, I187, M283, I327, A331, Y394, F397, and L439) and the methyl group of the side chains of Thr323 and Thr395 clustered in the surroundings of the aromatic ring to stabilize and recognize an a-methyl cinnamic acid. A model of AnFDC binding to $c c \mathrm{MA}$ was created using molecular operating environment (MOE) software. In the constructed FDC-ccMAbinding model, it was observed that Y394 formed a hydrogen bond with the carboxylate opp. $_{\text {. }}$ group, which is opposite the carboxylate react. $_{\text {group of }} c c \mathrm{MA}$.

ccMA decarboxylation reactions were assumed to be facilitated by an amino-acid substitution at the FDC substrate-binding site. This substitution would result in the capture of the carboxylate $e_{o p}$. group while those amino-acid residues (R173 and E282) involved in the decarboxylation reaction would be conserved. We generated single-substitution mutants in silico and arranged them in order of affinity improvement factor against $c c \mathrm{MA}$, after which we produced the top 25 designs of $A n$ FDC mutants and investigated the activity of those mutants (Fig. 2b). For measurement of enzymatic activity, we coexpressed UbiX derived from $E$. coli together with $A n F D C$ to synthesize prFMN. As a result of in vivo screening, wild-type (WT) the enzymatic activity of $A n$ FDCs was capable of producing 1,3-butadiene, indicating that WT AnFDCs can recognize ccMA as a substrate and mediate the decarboxylation of both $c c \mathrm{MA}$ and pentadienoic acid (PA; a ccMA-decarboxylated compound). We observed marked improvement in the activity of the three types of T395-substituted AnFDC mutants, and AnFDC T395Q, in particular, yielded a 109 \pm 5.26 -fold increase in butadiene production compared to that of the WT. An interaction formed between the substituted aminoacid residues $(\mathrm{H}, \mathrm{Q}, \mathrm{N})$ and the $c c \mathrm{MA}$ carboxylate $\mathrm{opp}_{\text {. group in the }}$ FDC mutant-ccMA binding model demonstrated this improved activity. Furthermore, the carboxyl $\mathrm{opp}_{\text {. }}$ group appeared to interact with Y394 and each of the substituted amino-acid residues (Supplementary Fig. 1). Taken together, these results showed that 


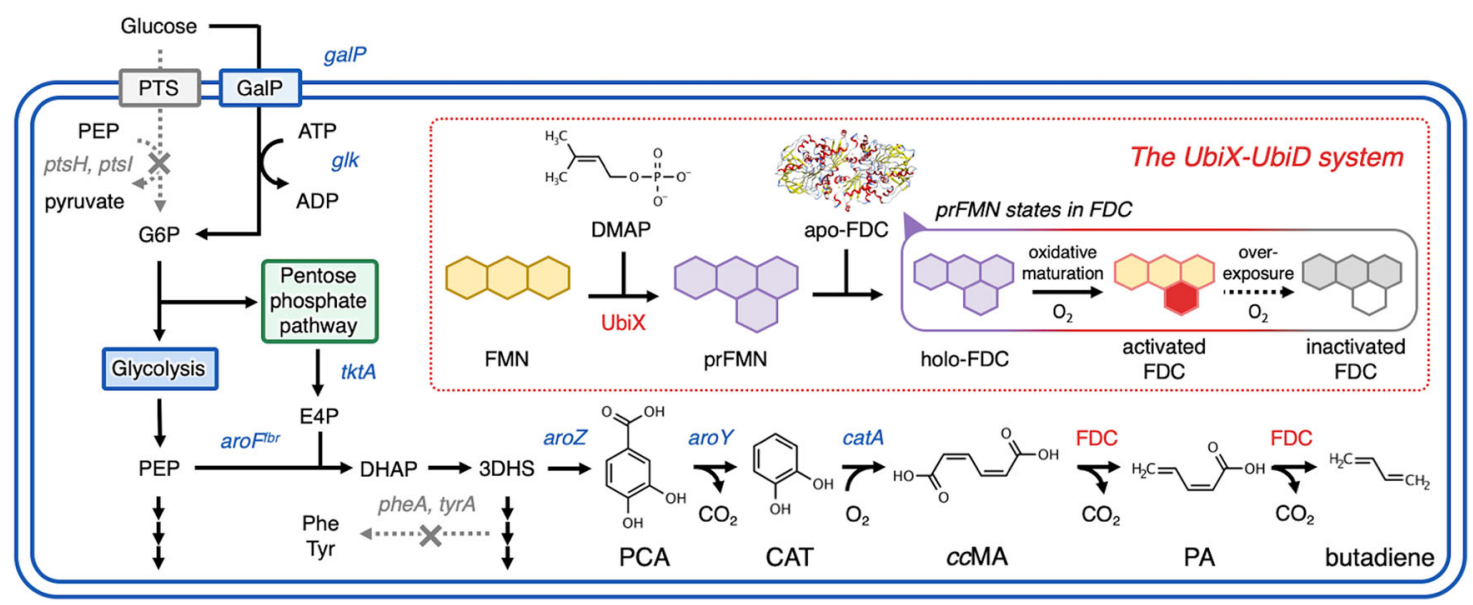

Fig. 1 Artificial metabolic pathway for the direct production of 1,3-butadiene from glucose using the UbiX-UbiD system. prFMN is biosynthesized via UbiX from both FMN and DMAP and then binds to apo-FDC. FDC requires exposure to oxygen for enzymatic activity, whereas overexposure to oxygen causes loss of activity. PEP phosphoenolpyruvate, PTS PEP-dependent phosphotransferase system, ATP adenosine triphosphate, ADP adenosine diphosphate, G6P glucose-6-phosphate, E4P erythrose-4-phosphate, DAHP 3-deoxy-D-heptulosonate-7-phosphate, 3DHS 3-dehydroshikimate, PCA protocatechuic acid, CAT catechol, ccMA cis,cis-muconate, PA (Z)-pentadienoate, Phe L-phenylalanine, Tyr L-tyrosine, FDC ferulic acid decarboxylase, FMN flavin mononucleotide, DMAP dimethylallyl phosphate, prFMN prenylated FMN.

a

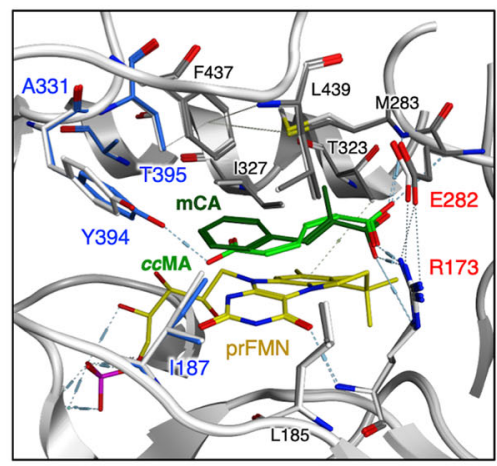

b

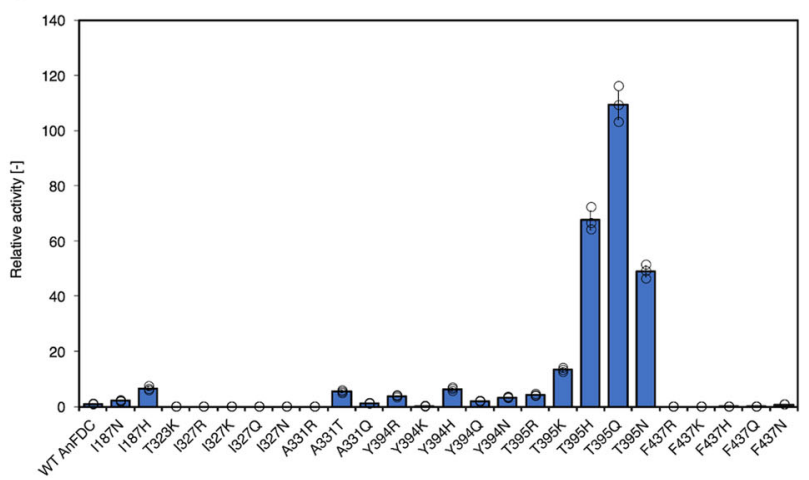

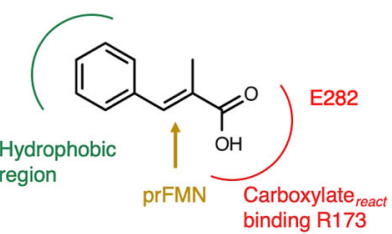

Carboxylate $_{o p p}$ binding pocket

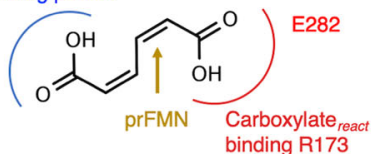

C

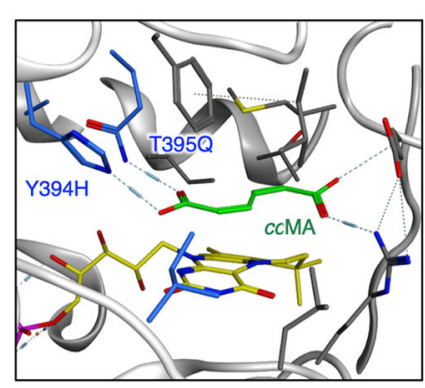

Fig. 2 Design of AnFDC for 1,3-butadiene production. a Overlay of the active site of AnFDC with bound $\alpha$-methyl cinnamic acid (dark green) from PDB:4ZA7, a model of ccMA (light green)-bound AnFDC with the lowest energy poses and a schematic design for ccMA. The negative hydrogen network and hydrophobic interactions are shown as dashed lines. b Relative decarboxylation activity of 25 AnFDC mutants designed for ccMA. The activity of WT AnFDC was defined as 1 . The data are presented as the means \pm SDs of three independent experiments $(n=3)$. c Simulated active site of the designed AnFDC Y394H:T395Q with bound ccMA. AnFDC ferulic acid decarboxylase derived from Aspergillus niger, mCA $\alpha$-methyl cinnamic acid, ccMA cis,cismuconic acid, SD standard deviation. Source data underlying Fig. $2 \mathrm{~b}$ are provided as a Source Data file.

the T395 substitution site is a critical position for $c c \mathrm{MA}-A n \mathrm{FDC}$ binding.

To further improve substrate specificity for $c c \mathrm{MA}$, we created a multiple-mutant library by combining these T395-substituted $A n$ FDC mutants and other single mutants that presented levels of activity higher than those of the WT (Supplementary Table 1). Compared with the WT AnFDC, the best-designed AnFDC Y394H:T395Q presented a $1002 \pm 35.6$-fold increase in 1,3-butadiene production from ccMA (Supplementary Table 2). The simulated model of AnFDC Y394H:T395Q and $c c M A$ showed 
a

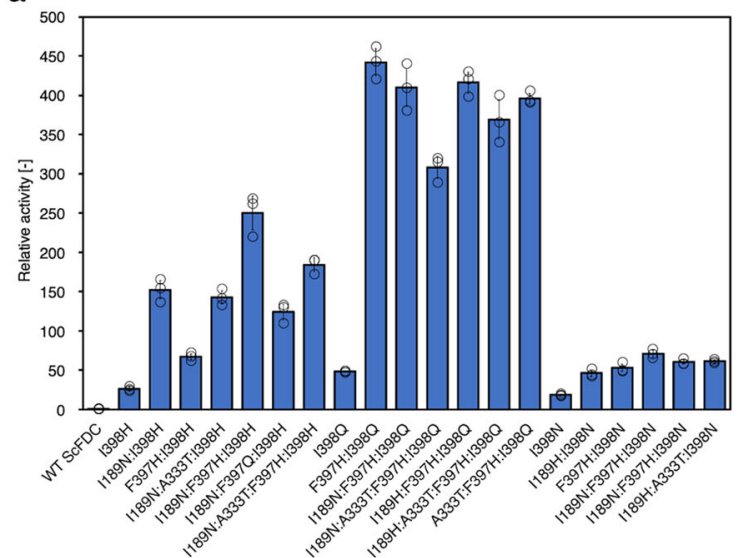

b

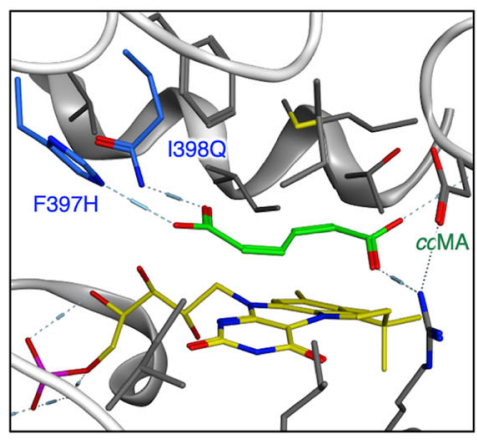

C

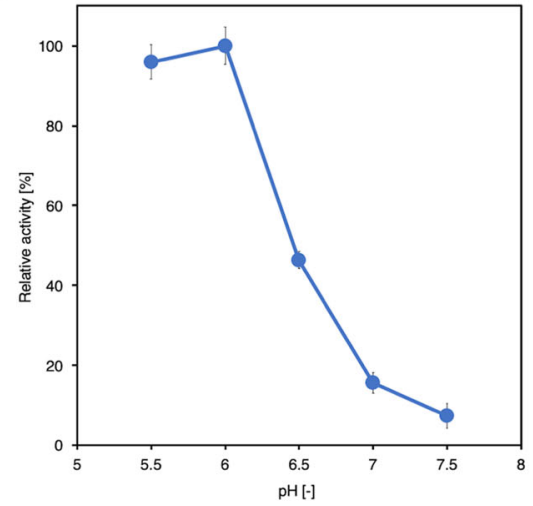

d

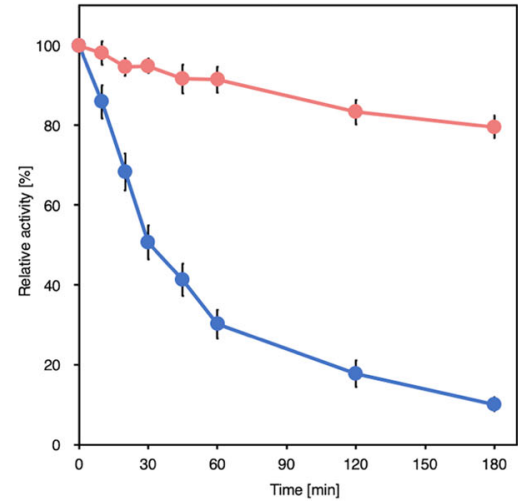

Fig. 3 Design of ScFDC for 1,3-butadiene production. a Relative decarboxylation activity of the top 20 ScFDCs designed for ccMA. The activity of WT AnFDC was defined as 1. b Simulated active site of the designed ScFDC F397H:I398Q with bound ccMA (based on PDB:4ZAC). The negative hydrogen network and hydrophobic interactions are shown as dashed lines. c pH dependence of the decarboxylation activity of the designed ScFDC F397H:I398Q for 1,3-butadiene. d Time course of activity of ScFDC F397H:I398Q under aerobic (blue) or oxygen-depleted (red) conditions. The activity was defined as $100 \%$ at 0 min after the incubation started. The data are presented as the means \pm SDs of three independent experiments $(n=3)$. ScFDC ferulic acid decarboxylase derived from Saccharomyces cerevisiae. SD standard deviation. Source data underlying Figs. 3a, 3c, and 3d are provided as a Source Data file.

that two substituted residues ( $\mathrm{H}$ and $\mathrm{Q})$ interact with the carboxylate $_{\text {opp. }}$ group of $c c \mathrm{MA}$, maintaining the interaction

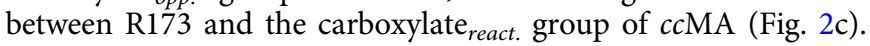
Taken together, these results suggested that the interaction between the FDC mutant and $c c \mathrm{MA}$ enables the efficient capture of ccMA, improving the enzymatic ability to produce 1,3butadiene.

Developing and characterization of ScFDC mutants by applying an $A \boldsymbol{n} F \mathrm{CD}$ mutant design. We developed ScFDC mutants by applying the good designs of the $A n \mathrm{FDC}$ mutants. The sequence homology between the AnFDC and ScFDC proteins was $48.4 \%$, and their substrate-binding sites have three differences; the amino-acid residues A331:Y394:T395 in AnFDC correspond to V334:F397:I398 in ScFDC (Supplementary Fig. 2). In addition to WT AnFDC, WT ScFDC also mediates ccMA decarboxylation (Fig. 3a). The best-designed ScFDC F397H:I398Q, to which the best-designed AnFDC Y394H:T395Q was applied, showed $441.9 \pm 17.2$-fold greater activity compared with that of WT ScFDC. The simulated model of ScFDC F397H:I398Q and $c c$ MA showed that two substituted residues interact with the carboxylate $_{\text {opp. }}$ group of $c c \mathrm{MA}$ (Fig. 3b). Compared with AnFDC Y394H: T395Q, ScFDC F397H:I398Q-mediated ccMA decarboxylation more efficiently as the $c c \mathrm{MA}$ concentration increased and ultimately presented a $1.61 \pm 0.35$-fold increase in enzymatic activity (Supplementary Fig. 3).
The $\mathrm{pH}$ dependence of the enzymatic activity of ScFDC F397H: I398 was analyzed. The optimal pH of ScFDC F397H:I398Q for ccMA decarboxylation was 6.0 , but the enzymatic activity significantly decreased at $\sim \mathrm{pH} 7.0$ (Fig. 3c).

Although AroY, which uses the same coenzyme that prFMN uses, requires exposure to oxygen to induce activity, it is known that overexposure to oxygen causes loss of enzyme activity ${ }^{29}$. Therefore, we also investigated the effects of oxygen on ScFDC F397H:I398Q. After incubation of ScFDC F397H:I398Q under aerobic or oxygen-depleted conditions, the $c c \mathrm{MA}$ was added and its enzyme activity was measured. The relationship between incubation time and the enzymatic activity of FDC is shown in Fig. 3d. The enzymatic activity of ScFDC F397H:I398Q at 0 min after incubation started was defined as $100 \%$. The ScFDC F397H: I398Q activity has a half-life of 30 min under aerobic conditions but decreased to $10.1 \pm 1.7 \%$ after $180 \mathrm{~min}$. On the other hand, $79.6 \pm 2.9 \%$ of the activity remained after $180 \mathrm{~min}$ under oxygendepleted conditions. These results indicated that the activity of FDC decreased due to continued oxygen exposure.

We investigated the substrate specificity of ScFDC F397H: I398Q using the $c c \mathrm{MA}$ isomers $c t \mathrm{MA}$ and $t \mathrm{MA}$. We observed that WT ScFDC recognized both $c c \mathrm{MA}$ isomers as substrates and converted them to butadiene via two decarboxylation reactions. Although ScFDC F397H:I398Q was designed for $c c$ MA, $45.8 \pm$ 4.2-fold (ctMA) and 12.0 \pm 1.4 -fold ( $t$ MA) enhancement of butadiene production by ScFDC F397H:I398Q was observed, compared with that of WT ScFDC. These findings demonstrated 
a

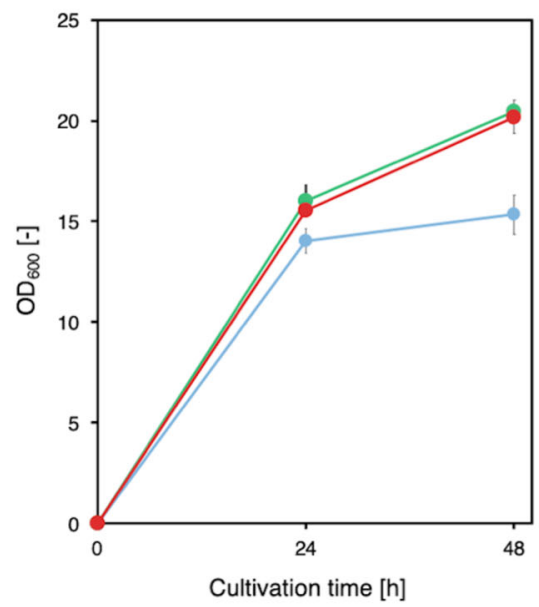

b

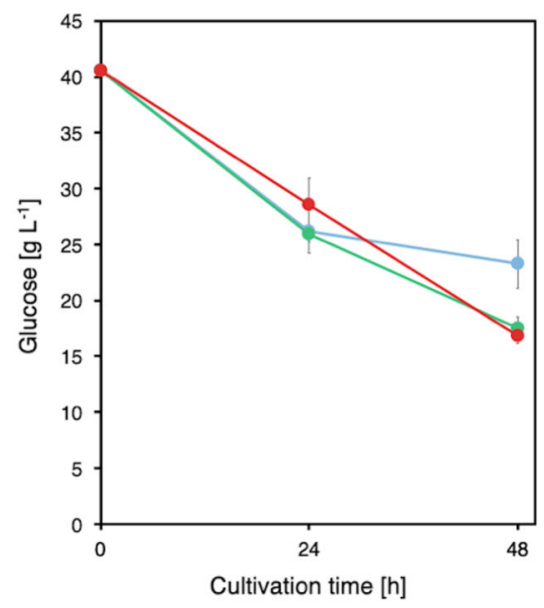

c

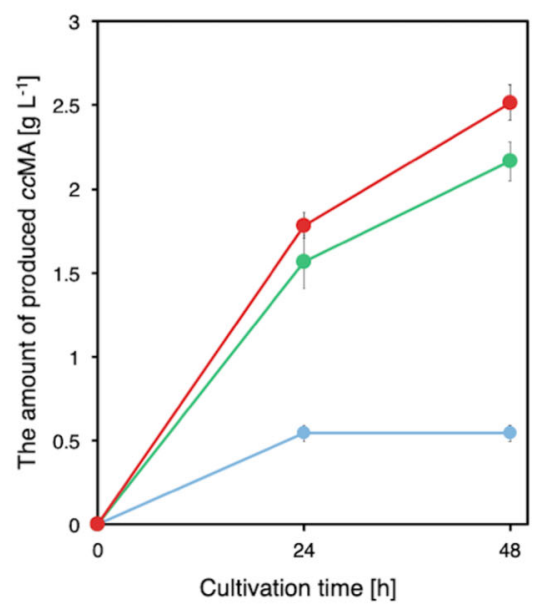

Fig. 4 Culture profiles of ccMA-producing CFB01, CFB11, and CFB21 strains. Time courses of a bacterial cell growth and $\mathbf{b}$ glucose consumption. c ccMA yield with cultivation time. Solid blue circles, CFB01; solid green triangles, CFB11; solid red squares, CFB21. The data are presented as the means \pm SDs of three independent experiments $(n=3)$. ccMA cis,cis-muconic acid, SD standard deviation. Source data are provided as a Source Data file.

that the mutated residues interact with the carboxyl group ${ }_{o p p}$ of $c c \mathrm{MA}$, promoting the capture of the carboxyl group $\mathrm{opp}_{\text {of }}$ ocMA isomers, and that ScFDC F397H:I398Q efficiently converted them to butadiene.

We investigated the substrate specificity of ScFDC F397H: I398Q for PA, an intermediate reaction that occurs when muconic acid undergoes a single decarboxylation. The results showed that the relative activity of ScFDC F397H:I398Q for WT ScFDC was $1.12 \pm 0.11$. A ScFDC F397H:I398Q and PA docking model is shown in Supplementary Fig. 4a. We confirmed that substituted F397H, I398Q, and a terminal alkene group of PA were separated. Furthermore, we investigated butadiene production from ccMA by combining WT ScFDC and ScFDC F397H: I398Q. The activity decreased to $56.3 \pm 7.6 \%$ when WT ScFDC and ScFDC F397H:I398Q were coexpressed compared to that when only ScFDC F397H:I398Q was expressed (Supplementary Fig. 4b). Based on these results, it was shown that ScFDC F397H: I398Q can recognize both $c c \mathrm{MA}$ and PA as substrates and can efficiently produce 1,3-butadiene from $c c$ MA by a double decarboxylation reaction; thus, only ScFDC F397H:I398Q was used for the butadiene production pathway to be introduced into E. coli.

Developing a $c c M A$-producing $E$. coli strain from glucose. To construct an artificial pathway through which 1,3-butadiene is produced from glucose, we developed an E. coli strain for $c c \mathrm{MA}$ production. In this study, we selected the pathway involving protocatechuic acid (PCA), the shortest pathway reported to be a $c c \mathrm{MA}$ production pathway, and conducted genome engineering for efficient $c c$ MA production (Fig. 1$)^{4}$. In this $c c$ MA pathway via PCA, $c c$ MA is produced from 3-dehydroshikimic acid (3DHS) by 3DHS dehydratase (aroZ), protocatechuic acid decarboxylase (aroY), and catechol dioxygenase (catA) in a three-step reaction. We selected aroZ derived from Bacillus thuringiensis, aro $Y$ from Klebsiella pneumoniae, and catA from Pseudomonas putida DOTT1E. For $c c M A$ production, these genes were incorporated into CFB01, CFB11, and CFB21; these genes constitute the basis of aromatic derivative-producing E. coli strains (Fig. 4) ${ }^{21,23}$. Cell growth and $c c \mathrm{MA}$ production of CFB01 nearly stopped after $24 \mathrm{~h}$ of culture, and $0.54 \pm 0.04 \mathrm{~g} \mathrm{~L}^{-1} c c \mathrm{MA}$ was produced. CFB1 1 and CFB21, the engineered strains designed for ccMA production, continued to grow and produce $c c \mathrm{MA}$ after $24 \mathrm{~h}$ of culture. After $48 \mathrm{~h}$ of culture, $2.17 \pm 0.09$ and $2.52 \pm 0.08 \mathrm{~g} \mathrm{~L}^{-1} c c \mathrm{MA}$ were produced by CFB01 and CFB02, respectively. Accumulations of intermediate metabolites, PCA, and catechol (CAT), were not observed. The highest yield of $c c \mathrm{MA}$ was $0.134 \pm 0.054 \mathrm{~mol}(\mathrm{~mol}$ glucose $)^{-1}$, which occurred from the CFB21 culture (Supplementary Table 3). Taken together, these results showed that we successfully generated a ccMA-producing E. coli strain (CFB21).

Constructing an artificial metabolic pathway for 1,3-butadiene production. By combining the $c c \mathrm{MA}$-producing E. coli strain from glucose with the ScFDC mutant, we constructed an artificial pathway through which 1,3-butadiene is produced from glucose. CFB22, which is CFB21 harboring UbiX, was cultured, and its ccMA production slightly decreased ( 10\%) (Supplementary Fig. 5).

Next, we developed a 1,3-butadiene-producing strain by introducing ScFDC into CFB21 and CFB22. These strains were cultured under aerobic conditions $(24 \mathrm{~h})$ and then packed culture (next $24 \mathrm{~h}$ ) conditions. CFB222, which is the CFB22 strain with ScFDC F397H:I398Q, grew and consumed glucose after packing (Supplementary Fig. 6). With this strain, $1.27 \pm 0.03 \mathrm{~g} \mathrm{~L}^{-1} c c \mathrm{MA}$ and $1.07 \pm 0.05 \mathrm{~g} \mathrm{~L}^{-1} \mathrm{PA}$ were produced (Fig. 5). A total of $41.4 \pm$ $2.82 \mathrm{mg} \mathrm{L}^{-1}$ of 1,3 -butadiene was produced by CFB222. Moreover, CFB212, a strain in which UbiX was not overexpressed, produced no 1,3-butadiene but produced a small amount of PA. These results showed that $U b i X$ overexpression is required for 1,3-butadiene production, although $\mathrm{UbiX}$ is inherently present in E. coli.

CFB222 was cultured in a closed 2-L medium bottle to examine the effects of oxygen conditions on butadiene production. FDC enzymatic activity requires oxygen for activation; 34 however, increased oxygen exposure leads to inactivation (Fig. 3d). In addition, cat $A$ requires oxygen to produce $c c \mathrm{MA}$ from CAT. Therefore, in this pathway for 1,3-butadiene production, aerobic conditions are suitable for the activation of FDC and for $c c M A$ production in the early stage of culture, whereas oxygen-depleted conditions are needed to maintain FDC activity. In a closed bottle, the gas phase was first applied under aerobic conditions and then gradually under oxygen-depleted conditions as the cells grew and consumed oxygen. In this packed culture, CFB222 was cultured at medium:air phase ratios of 1:5, 1:10, and 1:20. Glucose consumption and cell growth increased with increasing medium: air phase ratio, although they stopped after $24 \mathrm{~h}$ of culture (Fig. 6a). Similarly, ccMA, PA, and butadiene yield also increased 
a

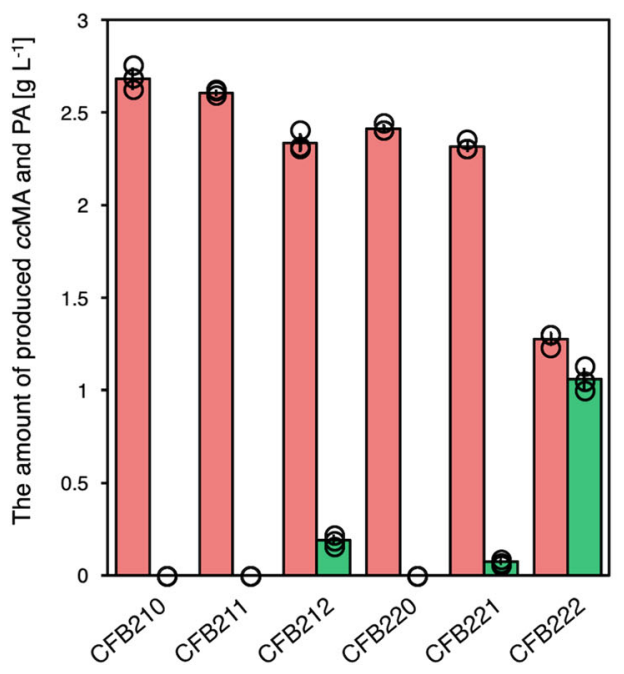

b

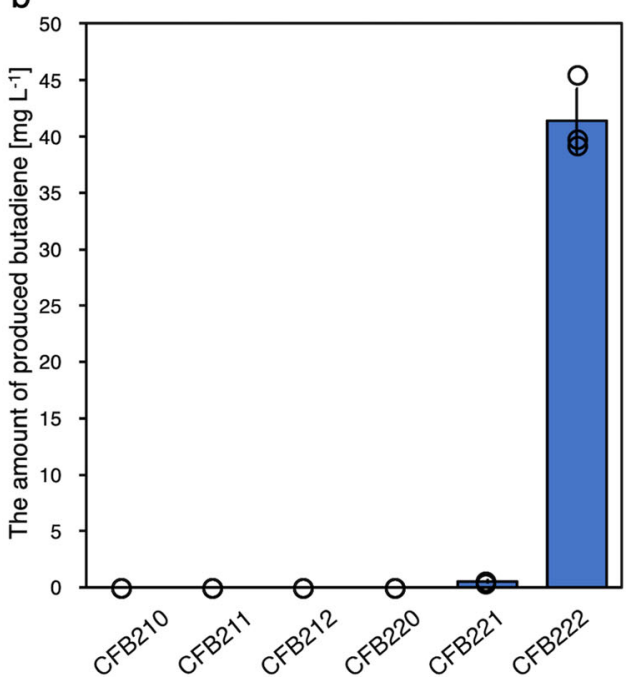

Fig. 5 Culture profiles of 1,3-butadiene-producing CFB210-CFB222 strains under aerobic conditions (24 h) and packed cultivation (another $24 \mathrm{~h}$ ) conditions. a ccMA (red) and PA (green) and $\mathbf{b}$ butadiene (blue) yields after $48 \mathrm{~h}$ of cultivation. The data are presented as the means \pm SDs of three independent experiments $(n=3)$. ccMA cis,cis-muconic acid, PA pentadienoic acid, SD standard deviation. Source data are provided as a Source Data file.

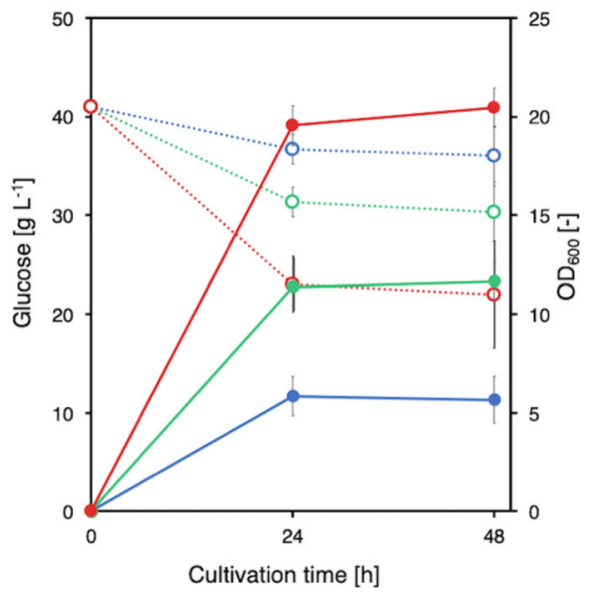

b

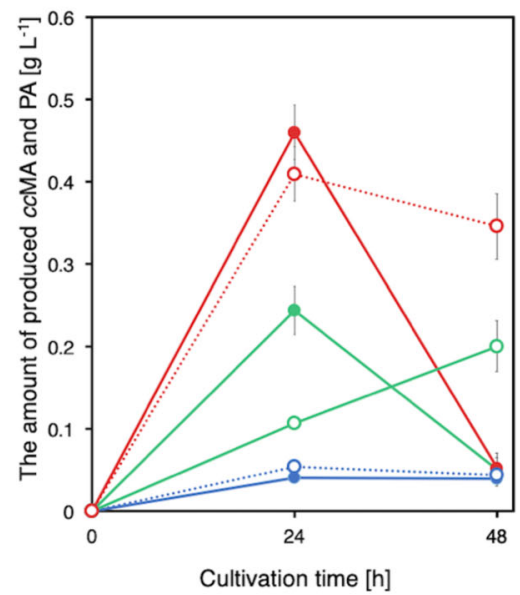

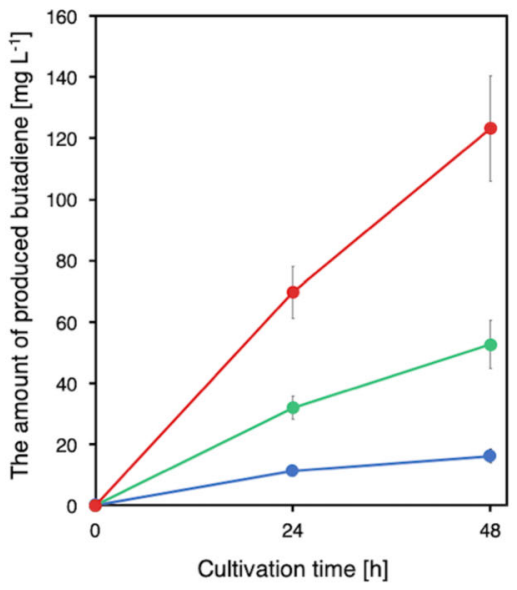

Fig. 6 Culture profiles of 1,3-butadiene-producing CFB222 strains under microaerobic conditions. a Time course of cell growth (solid circles, solid lines) and glucose consumption (open circles, dashed lines). The medium:air phase ratios were 1:5 (blue), 1:10 (green), and 1:20 (red). b Time courses of ccMA (solid circles, solid lines) and PA (open circles, dashed lines) produced. The medium:air phase ratios were 1:5 (blue), 1:10 (green), and 1:20 (red). c 1,3Butadiene yield with cultivation time. The medium:air phase ratios were 1:5 (solid blue), 1:10 (solid green), and 1:20 (solid red). The data are presented as the means \pm SDs of three independent experiments $(n=3)$. ccMA cis,cis-muconic acid, PA pentadienoic acid, SD standard deviation. Source data are provided as a Source Data file.

with an increase in the medium:air phase ratio (Figs. $6 \mathrm{~b}$ and $6 \mathrm{c}$ ). These results suggested that balancing aerobic and oxygendepleted conditions in culture is an important factor for 1,3butadiene production. Culturing (during which the $\mathrm{pH}$ was adjusted) was subsequently performed to examine the effects of $\mathrm{pH}$ on butadiene production (Supplementary Fig. 7). Butadiene production was higher at a low $\mathrm{pH}$ than at a $\mathrm{pH}$ of approximately 7.0, which is optimal for E. coli growth.

Based on the mass spectrum of standard 1,3-butadiene $(\mathrm{m} / \mathrm{z}=$ 50-54), the percentage of 1,3-butadiene isotopes produced was calculated (Supplementary Fig. 8). The main peak of 1,3-butadiene produced from natural glucose occurred at $\mathrm{m} / \mathrm{z}=$ 54 (Supplementary Fig. 9). With [U-13 C] glucose, a specific peak of $\left[{ }^{13} \mathrm{C}_{4}\right]$ 1,3-butadiene at $\mathrm{m} / \mathrm{z}=58(>70 \%)$ was observed, and peaks of $\left[{ }^{13} \mathrm{C}_{0-3}\right]$ 1,3-butadiene were also detected (Supplementary Fig. 10). It was suggested that $\left[{ }^{13} \mathrm{C}_{0-3}\right] 1,3$-butadiene was produced from $\left[\mathrm{U}_{-}{ }^{13} \mathrm{C}\right]$ glucose and carbohydrates in a yeast extract of the medium. When the yeast extract in the medium was reduced, the specific peak derived from $\left[{ }^{13} \mathrm{C}_{4}\right]$ 1,3-butadiene increased. Finally, in the absence of a yeast extract, $\left[{ }^{13} \mathrm{C}_{4}\right]$ 1,3-butadiene was predominant $(>95 \%)$. Taken together, these results indicated that 1,3-butadiene was produced mainly from glucose.

Production of 1,3-butadiene from glucose in a jar fermenter. Jar fermentation was performed under three different dissolved oxygen (DO) conditions at a $\mathrm{pH}$ of 7.0 (Supplementary Fig. 11). Under aerobic conditions (Supplementary Fig. 11a-c), the concentration of $c c \mathrm{MA}$ reached $3.28 \pm 0.29 \mathrm{~g} \mathrm{~L}^{-1}$ at $48 \mathrm{~h}$. Although the production of 1,3-butadiene stopped at $60 \mathrm{~h}, 123 \pm 8 \mathrm{mg} \mathrm{L}^{-1}$ 1,3 -butadiene was produced at $96 \mathrm{~h}$. Under microaerobic conditions (Supplementary Fig. 11d-f), the culture profile was almost the 
a

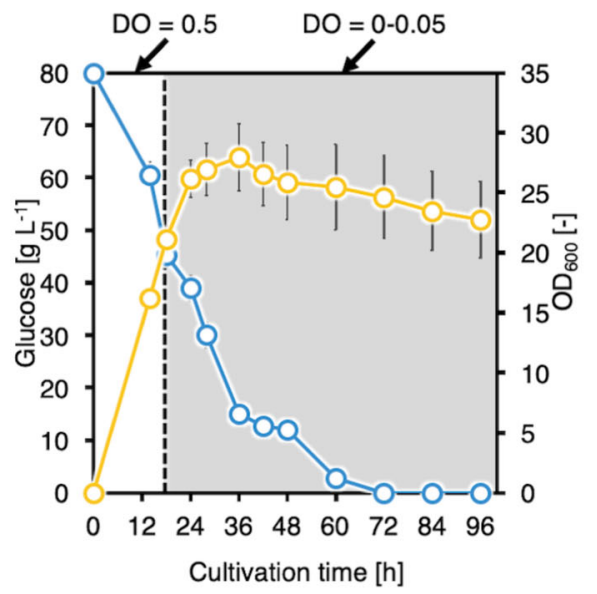

b

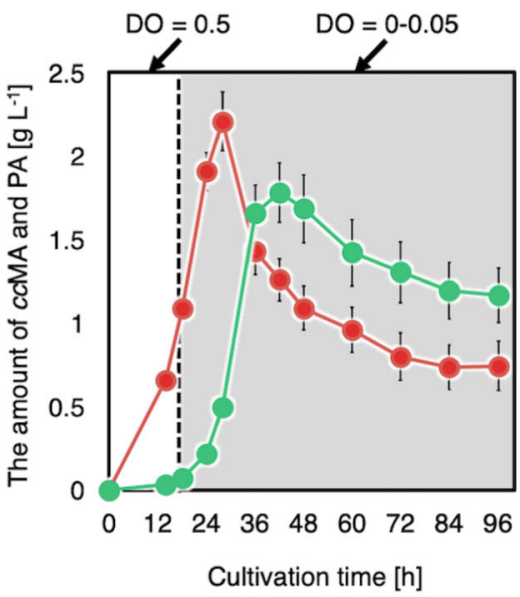

C

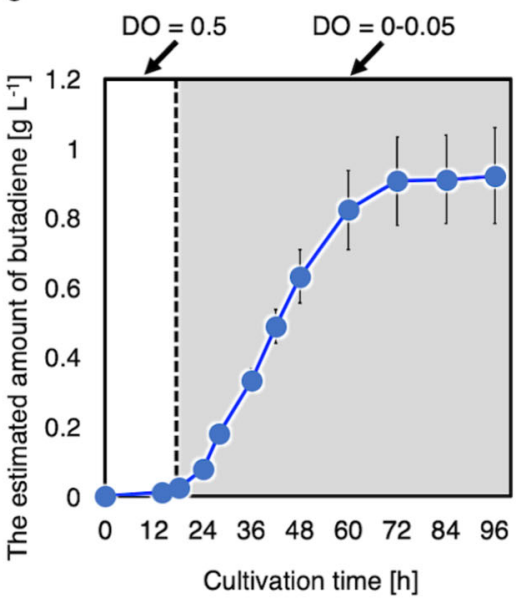

Fig. 7 Batch culture of CFB222 in a 1-L jar fermenter under DO-switching conditions at a pH of 6.0. Time courses of a bacterial cell growth (open yellow circles) and glucose concentration (open blue circles), b ccMA (solid red squares) and PA (solid green triangles) yields, and c estimated butadiene yield (solid blue circles). The data are presented as the means \pm SDs of three independent experiments $(n=3)$. DO dissolved oxygen, $c c M A$ cis, cis-muconic acid, PA pentadienoic acid, SD standard deviation. Source data are provided as a Source Data file.

same as that under aerobic conditions. These results suggested that a sufficient amount of $c c \mathrm{MA}$ was supplied for butadiene production even under microaerobic conditions. Under DO-shifted conditions (from aerobic to microaerobic conditions at $18 \mathrm{~h}$ ) (Supplementary Fig. $11 \mathrm{~g}-\mathrm{i}$ ), 1,3-butadiene production increased to $260 \pm 20 \mathrm{mg} \mathrm{L}^{-1}$ compared with that of the two above-mentioned conditions, although intermediate compounds $\left(3.0 \pm 0.27 \mathrm{~g} \mathrm{~L}^{-1}\right.$ ccMA and $\left.1.5 \pm 0.16 \mathrm{~g} \mathrm{~L}^{-1} \mathrm{PA}\right)$ accumulated. Therefore, we noted that switching from aerobic to microaerobic conditions is required when aiming to increase butadiene production.

Next, CFB222 fermentation under DO-shifted conditions (from aerobic to microaerobic conditions at $18 \mathrm{~h}$ ) at a $\mathrm{pH}$ of 6.0 was performed (Fig. 7). Glucose consumption and cell growth were slower than they were at a $\mathrm{pH}$ of 7.0 (Supplementary Fig. $11 \mathrm{~g}$ ). Although the $\mathrm{OD}_{600}$ value at $18 \mathrm{~h}$ was $27.3 \pm 1.3$ and reached $28.9 \pm 1.9$ at a $\mathrm{pH}$ of 7.0 , the $\mathrm{OD}_{600}$ value at $18 \mathrm{~h}$ was $21.2 \pm 0.8$ and reached $28.0 \pm 0.7$ at a $\mathrm{pH}$ of 6.0 . In addition, 1,3-butadiene was continually produced until $96 \mathrm{~h}$. Although $0.74 \pm 0.13 \mathrm{~g} \mathrm{~L}^{-1} c c \mathrm{MA}$ and $1.17 \pm 0.18 \mathrm{~g} \mathrm{~L}^{-1} \mathrm{PA}$ accumulated at the end of the culture, $0.91 \pm 0.09 \mathrm{~g} \mathrm{~L}^{-1}$ 1,3-butadiene was produced. Therefore, culturing at a $\mathrm{pH}$ of 6.0 resulted in higher butadiene production than did culturing at a $\mathrm{pH}$ of 7.0.

To further increase butadiene production, DO-stat fed-batch fermentation was performed (Fig. 8). Cell growth increased, and the maximum $\mathrm{OD}_{600}$ reached $35.0 \pm 3.2$. 1,3-Butadiene was produced until the end of the culture, although $1.37 \pm 0.06 \mathrm{~g} \mathrm{~L}^{-1} c c \mathrm{MA}$ and $2.33 \pm 0.41 \mathrm{~g} \mathrm{~L}^{-1} \mathrm{PA}$ accumulated. In total, $2.13 \pm 0.17 \mathrm{~g} \mathrm{~L}^{-1}$ 1,3 -butadiene was ultimately produced from glucose at $96 \mathrm{~h}$. We succeeded in increasing 1,3-butadiene production significantly via DO-stat fed-batch fermentation by changing the level of DO at a low $\mathrm{pH}$.

\section{Discussion}

1,3-Butadiene is a $\mathrm{C} 4$ diene and one of the most valuable industrial compounds. In this study, based on a rational design strategy of protein mutagenesis for developing target enzymatic functions, we constructed the artificial metabolic pathway for direct 1,3-butadiene production from glucose, which involves an entirely biological process.

Multiple classes of enzymes can produce biochemical compounds that have a terminal double bond. Fatty acid and fatty aldehyde decarboxylases have widespread substrate specificities and produce
C4-C20 terminal alkenes, which are important for sustainable fuel compounds ${ }^{44}$. Some dehydratases can also produce alkenes and acrylic acids by dehydration of the near-terminal hydroxyl group of substrates ${ }^{45,46}$. In a recent study, a class of enzymes was discovered: the biochemical reactions of these enzymes result in the formation of a terminal double bond, releasing ammonia, and formaldehyde ${ }^{47,48}$. The FDC used in this study, which contains prFMN biosynthesized by prenyltransferases, can catalyze the decarboxylation of $\alpha, \beta$-unsaturated carboxylic acids.

Computer-aided rational enzyme design can help improve the desired functions of target proteins, such as thermostability, enzymatic activity, and substrate specificity. With the evolution of computer simulation technology, many studies of enzyme development based on calculated parameter changes of substrate-docked enzyme modeling before and after in silico mutation have been reported $^{49-54}$. In this study, the best design, AnFDC Y394H:T395Q, achieved a $1002 \pm 35$.6-fold improvement in butadiene-producing activity compared with that of WT $A n$ FDC. The computersimulated model of AnFDC Y394H:T395Q shows that Y394H and T395Q interact with the carboxyl ${ }_{o p p}$ group of $c c \mathrm{MA}$ while maintaining the interaction between $\mathrm{R} 173$ and the carboxyl ${ }_{\text {react }}$ group. In addition, ScFDC F397H:I398Q, to which AnFDC Y394H:T395Q was applied, presented a $441.9 \pm 17.2$-fold increase in activity compared with that of WT ScFDC. Similarly, ScFDC F397H:I398Q also interacted with $c c \mathrm{MA}$, suggesting that this interaction altered substrate specificity and thereby improved the affinity of the FDC mutant for ccMA and increased enzymatic activity.

The optimal $\mathrm{pH}$ for the $c c \mathrm{MA}$ decarboxylation reaction involving ScFDC F397H:I398Q is 6.0, with a substantial decrease in activity at $\sim \mathrm{pH} 7.0$. In contrast, the optimal $\mathrm{pH}$ for the decarboxylation activity of WT ScFDC against the natural substrate cinnamic acid was 7.0 , and the enzymes could maintain $>90 \%$ of its activity at the $\mathrm{pH}$ range of $6.0-7.0^{55}$. Considering a $\mathrm{pKa}$ of the substituted His residue of $\sim 6.0$, we assumed that the protonated form of the imidazole side chain of His is required for efficient binding of $c c$ MA to ScFDC F397H:I398Q. AroY, an enzyme of the UbiD family, contains prFMN and is known to catalyze the decarboxylation of protocatecuate. The enzymatic activity of UbiD species requires oxygen to form the active prFMNiminium species $^{30,35}$, however, increased oxygen exposure leads to decrease the activity of some Ibid-Family members such as AroY ${ }^{29}$. It is reported that oxidative maturation of prFMN is required for FDC 
a

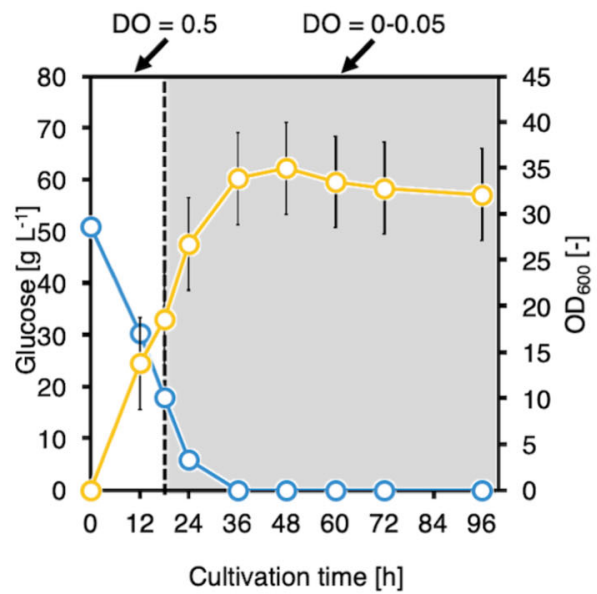

b

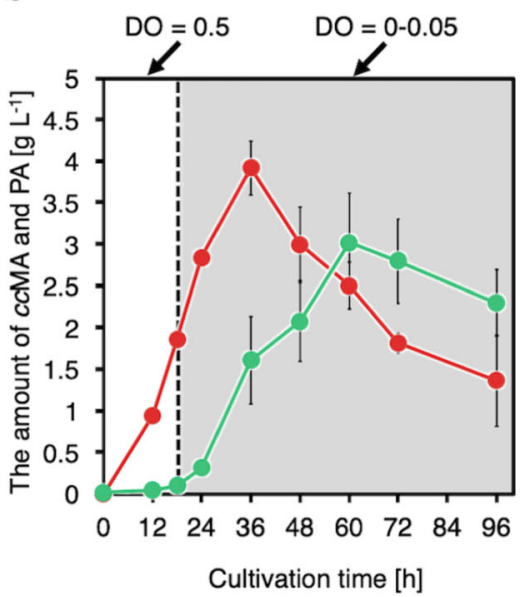

C

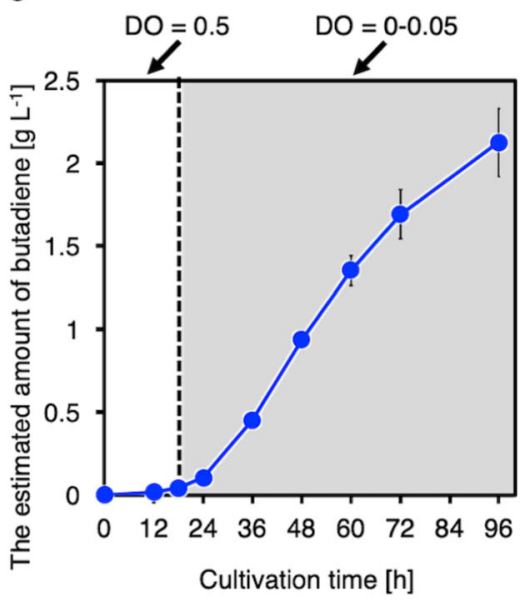

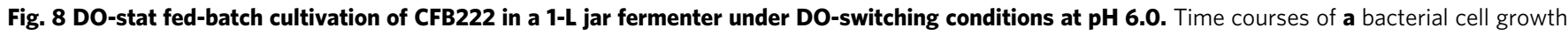
(open yellow circles) and glucose concentration (open blue circles), b ccMA (solid red squares) and PA (solid green triangles) yields, and c estimated butadiene yield (solid blue circles). The data are presented as the means \pm SDs of three independent experiments ( $n=3$ ). DO dissolved oxygen, $c c M A$ cis, cis-muconic acid, PA pentadienoic acid, SD standard deviation. Source data are provided as a Source Data file.

activity $^{34}$, although no oxygen sensitivity of the active holoenzyme has been reported. The oxygen sensitivity of purified ScFDC F397H:I398Q was measured in vitro, and a decrease of enzymatic activity of FDC under aerobic conditions was observed. To produce butadiene from $c c \mathrm{MA}$, the two decarboxylation reactions by ScFDC $\mathrm{F} 397 \mathrm{H}: \mathrm{I} 398 \mathrm{Q}$ are required, thus butadiene production may decrease drastically under aerobic conditions. Perhaps, the conformational change induced by ScFDC F397H:I398Q mutations in this study may lead to $\mathrm{O}_{2}$ sensitivity not observed for the WT ScFDC. The difference in decarboxylase activity between AnFDC Y394H:T395Q and ScFDC F397H:I398Q may depend on the differences in the ratio of the active/inactive prFMN contained ${ }^{25,27,34}$. ScFDC F397: I398Q activity for PA was largely unchanged compared with that of the WT (1.12 \pm 0.11 -fold increase). The PA molecule is smaller than muconic acid is. Therefore, we assumed that when PA forms a reactive binding state, it is less likely to interact with the substituted amino-acid residue F397H:I398Q and would not affect the affinity for PA. When WT ScFDC and ScFDC F397H:I398Q coexisted, the activity on $c c$ MA decreased $(56.3 \pm 7.6 \%)$ compared with that when only ScFDC F397H:I398Q was present. Although the activity for PA was virtually the same for WT ScFDC and ScFDC F397H:I398Q, ScFDC F397H:I398Q presented extremely high activity for $c c \mathrm{MA}$. Therefore, we assume that the first stage of the reaction from $c c \mathrm{MA}$ to PA decreased when WT ScFDC and ScFDC F397H:I398Q coexisted compared with that when only ScFDC F397H:I398Q was expressed.

We constructed the artificial metabolic pathway for 1,3-butadiene from glucose by combining a $c c$ MA-producing pathway with the decarboxylation of $c c$ MA by FDC. The butadieneproducing reaction in this study involves a two-step reaction that uses $c c \mathrm{MA}$ as an initial substrate, with PA generated from $c c \mathrm{MA}$ (first step) and butadiene generated from PA (second step) by decarboxylation reactions. The standard Gibbs free energy of the reaction is $\Delta \mathrm{G}^{0^{\prime}}=-14.2 \mathrm{~kJ} \mathrm{~mol}^{-1}$ for both reactions, making it thermodynamically ${ }^{56-58}$. At room temperature, butadiene is gaseous and insoluble in water; thus, the second step of the reaction is associated with a strong positive value. This reaction is therefore considered extremely useful as an artificial metabolic pathway for butadiene production.

Oxygen is required for prFMN activation and $c c \mathrm{MA}$ synthesis by cat $A$, whereas oxygen-depleted conditions are needed to maintain FDC activity. This was verified by the change in butadiene yield in an experiment in which packed bottles with different medium:air phase ratios were used. These results prompted us to change the DO conditions from being aerobic to microaerobic during culturing, and as a result, we successfully increased the butadiene yield. AroY, which generates CAT from PCA in the ccMA production pathway, is an enzyme that associates with prFMN as a coenzyme; therefore, we assumed that AroY activity would be affected by UbiX expression in the same way as FDC was. However, in vitro culturing of $c c \mathrm{MA}$-producing bacteria did not result in an increase in $c c$ MA production regardless of UbiX expression. This may have occurred because AroY was sufficiently active to produce $c c \mathrm{MA}$ owing to prFMN produced by endogenous UbiX. Although it is possible that AroY activity is reduced by oxygen, ccMA was produced by AroY and also by CatA in an experiment in which test tubes and jars were used under aerobic conditions, demonstrating that AroY has sufficient activity under these experimental conditions.

Jar fermentation at a relatively low $\mathrm{pH}$ increased 1,3-butadiene production. We propose two possible explanations for this. (1) The cell growth rate was lower at a $\mathrm{pH}$ of 6.0 than at a $\mathrm{pH}$ of 7.0, and proliferation of its growth continued after the conditions were shifted to microaerobic conditions. Therefore, we assume that an active form of the ScFDC mutant was produced that increased butadiene production. (2) The calculated $p K a$ values are 4.89 for $\mathrm{PA}$ and 3.87 and 4.65 for $c c \mathrm{MA}$, indicating that the proportion of PA and $c c$ MA protonated forms increases under low $\mathrm{pH}$ conditions. The protonated forms of $c c \mathrm{MA}$ and PA can reenter cells in a medium, which may promote the conversion to butadiene. The necessity of switching culture conditions from aerobic to microaerobic conditions and the requirement of a low $\mathrm{pH}$ have been shown previously. We therefore applied this knowledge to DO-stat-fed-batch cultures and thereby successfully increased butadiene production.

Two decarboxylation reactions are required for 1,3-butadiene production; that is, two moles of $\mathrm{CO}_{2}$ are lost, which results in a low carbon yield. Several recent studies on direct $\mathrm{CO}_{2}$ fixation involving the use of $E$. coli have been reported ${ }^{59,60}$. When this technique is applied to 1,3-butadiene-producing strains to fix $\mathrm{CO}_{2}$, increased carbon yield from 1,3-butadiene production is expected.

The intermediate substrates $c c \mathrm{MA}$ and PA remained in the medium at the end of fermentation. This means that the activity 
of the developed ScFDC mutant is still insufficient. Therefore, we hypothesize that butadiene production may be substantially increased when a mutant with increased affinity for PA is used and when that mutant is combined with ScFDC F397H:I398Q, as developed in this study. FDC mutant enzyme activity depends on the $\mathrm{pH}$, of which the optimum is 6.0 , and enzymatic activity may be considerably reduced when the E. coli intracellular $\mathrm{pH}$ is $6.8-7.5^{61}$. Therefore, we assumed that changing the optimal $\mathrm{pH}$ of FDC mutants to $6.8-7.5$ would promote FDC activity in E. coli and butadiene production.

Based on the rational enzyme design of the substrate-binding site, FDC was tailored to recognize the unnatural substrate $c c \mathrm{MA}$ and efficiently produce 1,3-butadiene via two decarboxylation reactions. By combining the $c c \mathrm{MA}$ production pathway and the decarboxylation of FDC, we generated an artificial metabolic pathway for direct 1,3-butadiene production. For 1,3-butadiene production, controlling the $\mathrm{DO}$ and $\mathrm{pH}$ is critical, and by optimizing these parameters, we achieved a dramatic increase in 1,3-butadiene yield. Because of the cellular toxicity of the intermediate compound PA, a tailored FDC mutant for PA should be developed to prevent growth inhibition and increase 1,3-butadiene production. The 1,3-butadiene yield from glucose can also be increased by selecting other $c c \mathrm{MA}$ pathways or further fine tuning of strains. By continued enzyme development to produce unnatural/nonbiological compounds that are currently made from petroleum, we can develop a circular bioeconomy in a sustainable society.

\section{Methods}

Chemicals and plasmid construction. We purchased $c c$ MA from Sigma-Aldrich (St. Louis, MO, USA), PA from Active Scientific Sdn Bhd (Kuala Lumpur, Malaysia), and 1,3-butadiene from Tokyo Chemical Industry (Tokyo, Japan). Supplementary Data 1 summarizes the strains and plasmids used in this study. We used E. coli NovaBlue-competent cells (Novagen, Cambridge, MA, USA) for gene cloning. We performed polymerase chain reaction (PCR) using KOD-Multi \& Epi DNA polymerase (Toyobo, Osaka, Japan) or PrimeSTAR Max (Takara, Shiga, Japan), and the primer pairs used are listed in Supplementary Data 2. We assembled each gene with the respective plasmid using NEBuilder HiFi DNA Assembly Master Mix (New England Biolabs, Ipswich, MA, USA).

We constructed pET-T7-AnFDC vectors as follows. Synthetic genes corresponding to AnFDC optimized for $E$. coli codon usage were obtained from a commercial source (Invitrogen, Carlsbad, CA, USA) (Supplementary Data 3). A fragment of the AnFDC gene was amplified via PCR using the synthetic gene AnFDC as the template in conjunction with the primer pair anfdc_T7_fw and anfdc_T7_rw. The plasmid fragment pSAK-Pt was also amplified via PCR using pSAK-Ptrc as the template in conjunction with the primer pair inv 12 trc fw and inv 12 trc rv. Each PCR fragment was conjugated to each other, and the obtained plasmid was named pET-T7AnFDC. We constructed pCOLA-T7-UbiX as follows. A fragment of the UbiX gene was amplified via PCR using E. coli as the template in conjunction with the primer pair anfdc_T7_fw and ubix_pcola_rv. The plasmid fragment pCOLA-T7 was also amplified via PCR using pCOLADuet-1 as a template in conjunction with the primer pair Inv_pcola_ubix_fw and Inv_pcola_ubix_rv. Each PCR fragment was conjugated to each other, and the obtained plasmid was named pCOLA-T7-UbiX. We constructed pET-T7-AnFDC-UbiX as follows. A fragment of the UbiX gene was amplified via PCR using pCOLA-T7-UbiX as a template in conjunction with the primer pair ubix_duet_fw and ubix_duet_rv. The plasmid fragment pET-T7-AnFDC was also amplified via PCR using pET-T7-AnFDC as a template in conjunction with the primer pair Inv_pet_duet_anfdc_fw and Inv_pet_duet_anfdc_rv. Each PCR fragment was conjugated to each other, and the obtained plasmid was named pET-T7-AnFDC-UbiX.

We constructed pZE12-Ptrc as follows. The $\operatorname{trc}$ promoter was amplified via PCR using pTrcHis B as a template in conjunction with the primer pair tre fw and trc_rv. The plasmid fragment pZE12 was also amplified via PCR using pZE12MCS as a template in conjunction with the primer pair inv_12_prom_fw and inv_12_prom_rv. Each PCR fragment was conjugated to each other, and the obtained plasmid was named pZE12-Ptrc. We constructed pZC12Am-Ptrc as follows. The colA gene was amplified via PCR using pCOLADuet-1 as a template in conjunction with the primer pair cola_fw and cola_rv. The plasmid fragment pZE12-Pt was also amplified via PCR using pZE12-Ptrc as a template in conjunction with the primer pair inv 12 ori fw and inv 12 ori rv. Each PCR fragment was conjugated to each other, and the obtained plasmid was named pZC12Am-Pt. We constructed pZC12Sp-Ptrc as follows. The gene fragment providing spectinomycin resistance was amplified via PCR using pTargetF as a template in conjunction with the primer pair sp_fw and sp_rv. The plasmid fragment pZC12-Pt was also amplified via PCR using pZC12Am-Ptrc as a template in conjunction with the primer pair inv_12_res_fw and inv_12_res_rv. Each PCR fragment was conjugated to each other, and the obtained plasmid was named pZC12Sp-Ptrc.

We constructed pSAK-aroFfbr-tktA as follows. A fragment of the tktA gene was amplified via PCR using E. coli K-12 MG1655 genomic DNA as a template in conjunction with the primer pair tkta_psti_fw and tkta_psti_rv. The amplified gene fragment was introduced into the PstI site of pS01. The resultant plasmid was

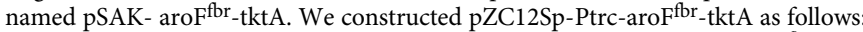
a fragment of the aroF-tktA gene was amplified via PCR using pSAK-aroFfbr-tktA as a template in conjunction with the primer pair fa $\operatorname{trc} f w$ and $\mathrm{fa} \operatorname{trc} r v$. The plasmid fragment pZC12Sp-Ptrc was also amplified via PCR using pZC12Sp-Ptrc as a template in conjunction with the primer pair inv_12_trc_fw and inv_12_trc_rv. Each PCR fragment was conjugated to each other, and the obtained plasmid was named pZC12Sp-Ptrc-aroFfbr-tktA.

We constructed pSAK-ZYC as follows: a fragment of the ZYC gene was amplified via PCR using pZA23-ZYC as a template in conjunction with the primer pair zyc_fw and zyc_rv. The plasmid fragment pSAK was also amplified via PCR using pSAK as a template in conjunction with the primer pair inv_laca_fw and inv_laca_rv. Each PCR fragment was conjugated to each other, and the obtained plasmid was named pSAK-ZYC. We constructed pSAK-ZYC-UbiX as follows. A fragment of the UbiX was amplified via PCR using pET-T7-AnFDC-UbiX as a template in conjunction with the primer pair ubix_smai_fw and ubix_smai_rv. The amplified fragment was introduced into the SmaI site of pSAK-ZYC, and the obtained plasmid was named pSAK-ZYC-UbiX.

We constructed pSAK-Ptrc as follows. A fragment of the trc promoter was amplified via PCR using pTrcHis B as a template in conjunction with the primer pair trc_ps_fw and trc_ps_rv. Fragments of the chloramphenicol resistance and SC101 origin genes were amplified via PCR using pZA33luc and pZS4Int-laci as templates with the primer pairs cmr_fw and cmr_rv and sc101_fw and sc101_rv, respectively Each PCR fragment was conjugated to each other, and the obtained plasmid was named pSAK-Ptrc. We constructed pSAK-Ptrc-ScFDC1 as follows. A fragment of the ScFDC1 gene was amplified via PCR using the synthetic gene ScFDC1 (Supplementary Data 4) as a template in conjunction with the primer pair scfdc_trc_fw and scfdc_trc_rv. The plasmid fragment pSAK-Pt was also amplified via PCR using pSAK-Ptrc as a template in conjunction with the primer pair inv 12 trc fw and inv 12 trc rv. Each PCR fragment was conjugated to each other and the obtained plasmid was named PSAK-Ptrc-ScFDC1. We constructed pET-T7ScFDC as follows. A fragment of the ScFDC1 gene was amplified via PCR using pSAK-Ptrc-ScFDC1 as a template in conjunction with the primer pair scfdc_T7_fw and scfdc_T7_rv. The plasmid fragment pET-T7 was also amplified via PCR using pET22b $(+)$ as a template in conjunction with the primer pair Inv_pet_T7_scfdc_fw and Inv_pet_T7_scfdc_rv. Each PCR fragment was conjugated to each other, and the obtained plasmid was named pET-T7-ScFDC. We constructed pET-T7-ScFDCScFDC F397H:I398Q as follows: a fragment of the ScFDC gene F397H:I398Q was amplified via PCR using pET-T7-ScFDC F397H:I398Q as a template in conjunction with the primer pair ScFDC F397H:I398Q_duet_fw and ScFDC F397H:

I398Q_duet_rv. The plasmid fragment pET-T7-ScFDC was also amplified via PCR using pET-T7-ScFDC as a template in conjunction with the primer pairs Inv_pet_duet_ScFDC F397H:I398Q_fw and Inv_pet_duet_ScFDC F397H:I398Q_rv. Each PCR fragment was conjugated to each other, and the obtained plasmid was named pET-T7-ScFDC-ScFDC F397H:I398Q.

We constructed pET-T7-ScFDC F397H:I398Q-His_tag as follows: a fragment of the ScFDC F397H:I398Q was amplified via PCR using pET-T7-ScFDC F397H: I398Q as a template in conjunction with the primer pair ScFDC His-tag fw and ScFDC His-tag_rv. The fragment was conjugated and the obtained plasmid was named pET-T7-ScFDC F397H:I398Q-His_tag.

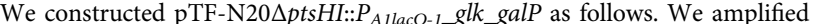
ptsHI-deficient gene fragments via PCR using E. coli K-12 MG1655 gDNA as a template in conjunction with the primer pairs ptshi_1300_fw and ptshi_1300_rv and ptshi_r300_fw and ptshi_r300_rv. We also amplified a $P_{\text {A1laco-1_glk_galP }}$ cassette via PCR using pCFTdeltain-GG as a template in conjunction with the primer pair lac_gk_gp_fw and lac_gk_gp_rv ${ }^{21}$. Moreover, we amplified the plasmid fragment $\mathrm{pTF}$ via PCR using pTargetF as a template in conjunction with the primer pair inv_ptf_fw and inv_ptf_rv. We assembled the three PCR fragments and named the obtained plasmid pTF- $\Delta$ ptsHI::P $P_{\text {Allaco-1 }} g l k$ galP. To introduce the

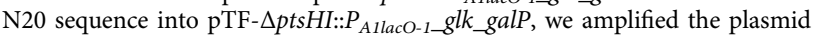

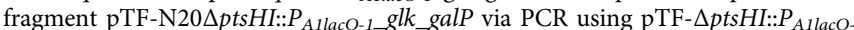
$1 \_g l k \_g a l P$ as a template in conjunction with the primer pair inv_n20_ptshi_fw and inv_n20_ptshi_fw. The resulting fragment self-assembled, and we named the

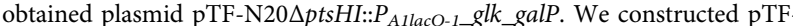
$\mathrm{N} 20 \Delta p h e A \Delta t y r A$ as follows. We amplified $p h e A$ - and tyrA-deficient gene fragments via PCR using E. coli K-12 MG1655 gDNA as a template in conjunction with the primer pairs phea_1300_fw and phea_1300_fw and tyra_r300_fw and tyra_r300_rv, respectively. We also amplified the plasmid fragment pTF via PCR using pTargetF as a template in conjunction with the primer pair inv_ptf fw and inv ptf_rv. We assembled the PCR fragments and named the obtained plasmid pTF- $\Delta$ pheA $\Delta t y r A$ To introduce the N20 sequence into pTF- $\Delta p h e A \Delta t y r A$, we amplified the plasmid fragment pTF- $\Delta$ pheA $\Delta$ tyrA via PCR using pTF- $\Delta p h e A \Delta t y r A$ as a template in conjunction with the primer pair inv_n20_phea_tyra_fw and inv_n20_phea_tyra_rv. The resultant fragment self-assembled, and we named the obtained plasmid pTF-N20 $\Delta$ pheA $\Delta$ tyrA. 
Chromosomal gene inactivation. Using the clustered, regularly interspaced, short palindromic repeat (CRISPR)/CRISPR-associated 9 (Cas9) two-plasmid system together with pTargetF and pCas, we inactivated the tandem gene set of pheA and tyra in the chromosome and replaced the tandem gene set of $p t s H$ and ptsI with the $P_{\text {Allaco-1_glk_galP cassette }}{ }^{18}$. To inactivate $p t s H$ and $p t s I$ and $p h e A$ and tyrA, we used pTF-N20 $\Delta$ ptsHI:: $P_{A 1 l a c O-1 \_g l k \_g a l P}$ and PTF-N20 $\Delta$ pheA $\Delta$ tyrA, respectively. We named these strains CFB1 and CFB2, respectively.

\section{Construction of the ccMA-FDC-binding model, docking simulation, and in}

silico mutagenesis. To obtain a model of $c c \mathrm{MA}$-bound $A n \mathrm{FDC}$, we replaced the binding substrate, $\alpha$-methyl cinnamic acid, of the substrate-bound crystallographic structure of $A n$ FDC (PDB:4ZA7) with $c c \mathrm{MA}$ via the 3D builder tool of MOE 2019.0101. The two models were then protonated using the protonate3D tool of $\mathrm{MOE}$ at a $\mathrm{pH}$ of 7 and a temperature of $300 \mathrm{~K}$ and then optimized by energy minimization using the AMBER10:extended Hückel theory (EHT) force field (gradient $=0.01 \mathrm{RMS} \mathrm{kcal} \mathrm{mol}^{-1} \mathrm{~A}^{-2}$ ). For docking simulation, the force field of AMBER10:EHT and the implicit solvation model of the reaction field (R-field 1:80; cutoff ${ }^{8,10}$ ) were selected. The docking simulations were carried out with the general dock tool of MOE, and the settings were as follows: site, ligand atoms; ligand, $c c \mathrm{MA}$; placement, triangle Matcher method and London $\Delta \mathrm{G}$ scoring; and refinement, induced fit and GBVI/WSA $\Delta \mathrm{G}$ scoring. The best scored configurations were selected for further analysis.

The residue scan tool of MOE was used for in silico mutation analysis of $c c \mathrm{MA}$ bound AnFDC, and the settings were as follows: residues, L185, I187, M283, T323, I327, A331, Y394, T395, F397, and L439; mutations, arginine, lysine, histidine, aspartic acid, glutamic acid, serine, threonine, asparagine, glutamine, tyrosine, and cysteine; site limit, 1 ; and affinity atoms, $c c \mathrm{MA}$. The effect of the mutation on the binding free energy $\left(\Delta \mathrm{G}_{\text {bind }}\right)$ between $c c \mathrm{MA}$ and the $A n \mathrm{FDC}$ mutants was calculated; the relative binding free affinity changes ( $\Delta$ affinity) between the $A n \mathrm{FDC}$ mutant $\left(\Delta \mathrm{G}_{\text {mutant }}\right) A n \mathrm{FDC}$ mutant $\left(\Delta \mathrm{G}_{W T}\right)$ were obtained from MOE. The models of multiple AnFDC mutants were generated with the protein builder tool of $\mathrm{MOE}$, and the models of $c c \mathrm{MA}$-bound AnFDC mutants were constructed as mentioned above.

To obtain a model of $c c$ MA-bound ScFDC F397H:I398Q, we first superimposed the generated model of $c c \mathrm{MA}$-bound $A n \mathrm{FDC}$ and the crystal structure of ScFDC (PDB:4ZAC) and removed AnFDC and AnFDC-binding prFMN. The $c c$ MA-bound WT ScFDC model was then constructed as mentioned above. Next, the WT ScFDC model was mutated with the protein builder tool of MOE such that ScFDC F397H: I398Q was generated. The models of $c c$ MA-bound ScFDC F397H:I398Q and PAbound ScFDC F397H:I398Q were constructed as mentioned above.

Construction and screening of AnFDC and ScFDC mutants. After calculating the change in affinity between the $A n \mathrm{FDC}$ mutants and $c c \mathrm{MA}$ in silico, we selected the top 25 designs on the basis of their affinity. To construct AnFDC mutants, we performed site-directed mutagenesis via inverse PCR. We amplified pET-T7AnFDC plasmids with the specific primer pair required for each mutation. The PCR products were subsequently self-ligated to construct mutated plasmids.

We precultured recombinant $E$. coli cells harboring FDC (WT or mutant $A n$ FDC) and UbiX in $0.5 \mathrm{~mL}$ of Luria-Bertani (LB) medium consisting of $100 \mu \mathrm{g}$ $\mathrm{mL}^{-1}$ ampicillin and $50 \mu \mathrm{gL}^{-1}$ kanamycin. After $3 \mathrm{~h}, 10-\mu \mathrm{L}$ precultures were added to $1 \mathrm{~mL}$ of screening medium consisting of $0.5 \mathrm{mM}$ disodium $c c \mathrm{MA}$ in a $10-\mathrm{mL}$ HS/GC-MS packed vial. The cultures were then incubated at $37^{\circ} \mathrm{C}$ in a shaker at $180 \mathrm{rpm}$. After $18 \mathrm{~h}$, using HS/GC-MS, we analyzed the 1,3-butadiene produced in the gas phase of the vial. The activity of AnFDC was calculated on the basis of the amount of produced butadiene. The screening medium consisted of (per liter) $20 \mathrm{~g}$ of lactose, $12 \mathrm{~g}$ of tryptone, $24 \mathrm{~g}$ of yeast extract, $12.5 \mathrm{~g}$ of $\mathrm{K}_{2} \mathrm{HPO}_{4}, 2.3 \mathrm{~g}$ of $\mathrm{KH}_{2} \mathrm{PO}_{4}$, antibiotics, and $0.1 \mathrm{mM}$ isopropanol as an internal standard. A disodium $c c \mathrm{MA}$ stock solution was prepared by mixing $c c \mathrm{MA}$ and $\mathrm{NaOH}$ (two equivalents) together. To further increase the enzymatic activity on $c c \mathrm{MA}$, we constructed a combinatorial mutant library, with AnFDC T395H, T395Q, or T395N used as template enzymes. The enzyme activities of these mutants were measured as mentioned above. ScFDC mutants were also constructed using pET-T7-ScFDC as a template, and these activities were measured as mentioned above.

Characterization of ScFDC F397H:I398Q. For analysis of the $\mathrm{pH}$ dependence of $S c$ FDC F397H:I398Q activity, we precultured recombinant $E$. coli cells harboring ScFDC F397H:I398Q and UbiX in $0.5 \mathrm{~mL}$ of LB medium with antibiotics. After $3 \mathrm{~h}$, we inoculated the cells into $30 \mathrm{~mL}$ of screening medium in a $300 \mathrm{~mL}$ conical flask and then sealed it with a parafilm. After $18 \mathrm{~h}$, we collected the cells by centrifugation. Afterward, we resuspended the cell pellet in $30 \mathrm{~mL}$ of B-PER bacterial cell lysis reagent (Thermo Fisher Scientific, Waltham, MA, USA). After it was shaken for $10 \mathrm{~min}$ at $180 \mathrm{rpm}$, we collected the cell lysate by centrifugation. We added $1 \mathrm{~mL}$ of the enzyme assay solution into a 10-mL HS/GC-MS vial and incubated it at $37^{\circ} \mathrm{C}$. After $18 \mathrm{~h}$, we analyzed the produced 1,3-butadiene in the gas phase of the vial via HS/GC-MS. The screening medium consisted of $1 / 10$ the volume of cell lysates, $0.5 \mathrm{mM}$ disodium $c c \mathrm{MA}$, and $10 \mathrm{mM}$ phosphate buffer. We used $0.1 \mathrm{mM}$ isopropanol as the internal standard.

For analyzing the time course of activity of ScFDC F397H:I398Q, hexa histidine-tagged ScFDC F397H:I398Q and UbiX was also coexpressed in E. coli.
ScFDC F397H:I398Q was purified with a His-tag attached to the C-terminal of ScFDC F397H:I398Q using a Ni-NTA column (His-Trap HP column $5 \mathrm{~mL}$, GE Healthcare Bio-Sciences Uppsala, Sweden) in $50 \mathrm{mM}$ phosphate buffer, $50 \mathrm{mM}$ $\mathrm{KCl}, \mathrm{pH} 7$, with wash and elution buffers supplemented with 10 and $250 \mathrm{mM}$ imidazole, respectively. Finally, purified His-tagged ScFDC F397H:I398Q was desalted into $20 \mathrm{mM}$ phosphate buffer containing $50 \mathrm{mM} \mathrm{KCl}$ (pH 6.0) on PD 10 Sepharose columns (GE Healthcare Bio-Sciences Uppsala, Sweden).

$0.1 \mathrm{vvm}$ (volume of gas per volume of liquid per minute) of nitrogen was bubbled through the $100 \mathrm{mM}$ potassium phosphate buffer consisting of $100 \mathrm{mM}$ $\mathrm{KCl}(\mathrm{pH} 6.0)$ for $30 \mathrm{~min}$. Then, the enzyme assay solution ( $1 \mu \mathrm{M} \mathrm{ScFDC} \mathrm{F397H:}$ $\mathrm{I} 398 \mathrm{Q}, 50 \mathrm{mM} \mathrm{KCL}$ and $50 \mathrm{mM}$ potassium phosphate buffer ( $\mathrm{pH} 6.0)$ ) was prepared. The solutions were incubated $0,10,20,30,45,60,120,180$ min under aerobic or oxygen-depleted conditions. After incubation, $8 \mathrm{~mL}$ of the enzyme assay solution was transferred into a 10-mL HS/GC-MS vial, and $8 \mu \mathrm{L}$ of 500 $\mathrm{mM}$ disodium $c c \mathrm{MA}$ stock was added. The final concentration of ccMA was 0.5 $\mathrm{mM}$. After packing and incubation for $18 \mathrm{~h}$ at $37^{\circ} \mathrm{C}$, we analyzed the produced 1,3-butadiene in the gas phase of the vial via HS/GC-MS. The activity of ScFDC F397H:I398Q was calculated on the basis of the amount of produced butadiene.

To analyze substrate specificity, $c t \mathrm{MA}$ was prepared from $c c \mathrm{MA}^{62}$. One milliliter of ultrapure water was added to $3.55 \mathrm{mg}$ of $c \mathrm{MAA}$, which was then heated at $80^{\circ} \mathrm{C}$ for $30 \mathrm{~min}$ at $2,000 \mathrm{rpm}$. After heating, $50 \mu \mathrm{L}$ of $1 \mathrm{M} \mathrm{NaOH}$ and ultrapure water were added to prepare a $20 \mathrm{mM}$ disodium $c t \mathrm{MA}$ stock solution. A $20 \mathrm{mM}$ disodium $c c \mathrm{MA}$ stock solution and a $20 \mathrm{mM}$ disodium $t \mathrm{tMA}$ stock solution were prepared by mixing $\mathrm{NaOH}$ (two equivalents). Sodium $(Z)-\mathrm{PA}(20 \mathrm{mM})$ was prepared by mixing one equivalent of $\mathrm{NaOH}$. The enzymatic activities of WT ScFDC and ScFDC F397H: I398Q against each substrate were measured as mentioned above.

For co-expression of WT ScFDC and ScFDC F397H:I398Q in E. coli, recombinant E. coli cells harboring pET-T7-ScFDC_WT-ScFDC F397H:I398Q and pCOLA-T7UbiX were cultured, and butadiene production was measured as described above.

All of the experiments with FDC were conducted under light-excluded conditions to prevent the inactivation of FDC by the light ${ }^{27}$.

Culture conditions. For $c c \mathrm{MA}$ and 1,3-butadiene production in $5 \mathrm{~mL}$ test tubescale cultures, we used a producing medium consisting of (per liter) $40 \mathrm{~g}$ of glucose, $12 \mathrm{~g}$ of tryptone, $24 \mathrm{~g}$ of yeast extract, $12.5 \mathrm{~g}$ of $\mathrm{K}_{2} \mathrm{HPO}_{4}, 2.3 \mathrm{~g}$ of $\mathrm{KH}_{2} \mathrm{PO}_{4}, 10 \mathrm{mg}$ of antibiotics, $100 \mathrm{mg}$ of L-phenylalanine, $40 \mathrm{mg}$ of L-tyrosine, and $40 \mathrm{mg}$ of L-tryptophan. We added $0.2 \mathrm{mM}$ isopropyl IPTG to induce protein expression and 10 $\mathrm{mM}$ sodium pyruvate to the medium to encourage bacterial growth in the initial phase. We added ampicillin, spectinomycin, and/or chloramphenicol to the medium up to final concentrations of 100,50 , and $15 \mu \mathrm{g} \mathrm{mL}^{-1}$, respectively. We seeded each preculture into $5 \mathrm{~mL}$ of this medium in a test tube, and we incubated the test tube-scale cultures at $37^{\circ} \mathrm{C}$ in a shaker at $180 \mathrm{rpm}$.

For 1,3-butadiene production, the resultant $E$. coli strains were cultured in a test tube for $24 \mathrm{~h}$, transferred into a $10-\mathrm{mL}$ of HS/GC-MS packed vial and cultured for an additional $24 \mathrm{~h}$. For 1,3-butadiene production under oxygen-depleted conditions, we added $1 \mathrm{~mL}$ of the seeded medium to a 10-mL HS/GC-MS packed vial, and the strain were incubated at $37^{\circ} \mathrm{C}$ in a shaker at $180 \mathrm{rpm}$. After culture, we analyzed the $1,3-$ butadiene produced in the gas phase of the vial HS/GC-MS.

We seeded each preculture into 100,200 , and $400 \mathrm{~mL}$ of the producing medium in a 2-L medium bottle. The cap had a tube that allowed sampling such that the media and gas phases could be collected with a syringe without opening the bottle. We incubated the oxygen-depleted cultures at $37^{\circ} \mathrm{C}$ in a shaker at $180 \mathrm{rpm}$. After measuring the $\mathrm{pH}$, we added a $10 \%$ ammonium solution to adjust the $\mathrm{pH}$ of the medium to 6.2, if needed. We used M9T medium for $\left[{ }^{13} \mathrm{C}_{4}\right] 1,3$-butadiene production in 1-mL HS/GC-MS vial-scale cultures with packing. M9T minimal medium consists of (per liter) $20 \mathrm{~g}$ of glucose, $12 \mathrm{~g}$ of tryptone, $0.5 \mathrm{~g}$ of NaCl, $17.1 \mathrm{~g}$ of $\mathrm{Na}_{2} \mathrm{HPO}_{4} \cdot 12 \mathrm{H}_{2} \mathrm{O}, 3 \mathrm{~g}$ of $\mathrm{KH}_{2} \mathrm{PO}_{4}, 2 \mathrm{~g}$ of $\mathrm{NH}_{4} \mathrm{Cl}, 246 \mathrm{mg}$ of $\mathrm{MgSO}_{4} \cdot 7 \mathrm{H}_{2} \mathrm{O}$, $14.7 \mathrm{mg}$ of $\mathrm{CaCl}_{2} \cdot 2 \mathrm{H}_{2} \mathrm{O}, 2.78 \mathrm{mg}$ of $\mathrm{FeSO}_{4} \cdot 7 \mathrm{H}_{2} \mathrm{O}, 10 \mathrm{mg}$ of thiamine hydrochloride, $100 \mathrm{mg}$ of L-phenylalanine, $40 \mathrm{mg}$ of L-tyrosine, $40 \mathrm{mg}$ of L-tryptophan, and $0.2 \mathrm{mM}$ IPTG. The percentage of butadiene isotopes was calculated according to the quintic equation. Each peak area distribution was created on the basis of its 1,3-butadiene standard (Supplementary Fig. 9).

$$
\left(\begin{array}{ccccc}
100 & 66.8 & 11.6 & 25.5 & 29 \\
0 & 100 & 66.8 & 11.6 & 25.5 \\
0 & 0 & 100 & 66.8 & 11.6 \\
0 & 0 & 0 & 100 & 66.8 \\
0 & 0 & 0 & 0 & 100
\end{array}\right)\left(\begin{array}{l}
a \\
b \\
c \\
d \\
e
\end{array}\right)=\left(\begin{array}{l}
A \\
B \\
C \\
D \\
E
\end{array}\right)
$$

$a, b, c, d, e=$ relative ratio of each isotope; $m / z=54,55,56,57,58$, respectively $A, B, C, D, E=$ each experimental peak area of $m / z=54,55,56,57,58$, respectively

$$
\text { Percentage of each isotope }=\frac{x}{a+b+c+d+e} \times 100 \%
$$

\section{$x=a, b, c, d$, or $e$}

We performed 1-L jar fermentation with a $400-\mathrm{mL}$ working volume. We added the preculture medium to the medium $(400 \mathrm{~mL})$ in a 1-L jar fermenter with an initial $\mathrm{OD}_{600}$ of 0.05 . The flow rate of air for fermentation was $100 \mathrm{~mL} \mathrm{~min}^{-1}$. To maintain the $\mathrm{pH}$ at 6.0 or 7.0 during culture, we added aqueous ammonia to the 
medium. We maintained the DO at $0.05-0.5 \mathrm{ppm}$ by automatically controlling the agitation speed from 200 to $800 \mathrm{rpm}$. The medium for jar fermentation consisted of (per liter) $12 \mathrm{~g}$ of tryptone, $24 \mathrm{~g}$ of yeast extract, $12.5 \mathrm{~g}$ of $\mathrm{K}_{2} \mathrm{HPO}_{4}, 2.3 \mathrm{~g}$ of $\mathrm{KH}_{2} \mathrm{PO}_{4}$, antibiotics, $100 \mathrm{mg}$ of L-phenylalanine, $40 \mathrm{mg}$ of L-tyrosine, $40 \mathrm{mg}$ of L-tryptophan, $0.2 \mathrm{mM}$ isopropyl $\beta$ - d-1-thiogalactopyranoside (IPTG) and $10 \mathrm{mM}$ sodium pyruvate. The initial glucose concentration was $80 \mathrm{~g} \mathrm{~L}^{-1}$ for batch fermentation. However, for DO-stat fed-batch fermentation, the initial glucose concentration was $50 \mathrm{~g} \mathrm{~L}^{-1}$. The feeding solution was added to the culture medium automatically with a pump when the DO was $>0.25 \mathrm{ppm}$ under microaerobic culture conditions (DO of $0 \sim 0.05 \mathrm{ppm}$, after $18 \mathrm{~h}$ ), and feeding was stopped when the DO was $<0.25 \mathrm{ppm}$. The feeding solution consisted of (per liter) $500 \mathrm{~g} \mathrm{~L}^{-1}$ glucose, $60 \mathrm{~g} \mathrm{~L}^{-1}$ tryptone, and $120 \mathrm{~g} \mathrm{~L}^{-1}$ yeast extract and was added up to $32 \mathrm{~mL}$. We collected the exhaust gas of the culture in HS/GC-MS vials.

Analytical methods. We monitored cell growth by measuring the $\mathrm{OD}_{600}$ with an UVmini-1240 spectrophotometer (Shimadzu, Kyoto, Japan). We also measured the concentration of glucose in the culture supernatant via a glucose CII test (Wako, Kyoto, Japan) according to the manufacturer's instructions.

We determined the concentrations of $c c \mathrm{MA}$ in the culture supernatants, which we separated from the culture broth by centrifugation at $15,000 \times g$ for $10 \mathrm{~min}$. To accomplish this, we used an organic acid analysis system (Shimadzu) comprising a highperformance liquid chromatography (HPLC) instrument equipped with two Shim-pack SPR-H columns, the first operating at $48^{\circ} \mathrm{C}$ and the second operating at $25^{\circ} \mathrm{C}$, with a flow rate of $0.8 \mathrm{~mL} \mathrm{~min}^{-1}$. We used a CDD-10A detector, $5 \mathrm{mM} \mathrm{p}$-toluenesulfonic acid (PTSA) as the mobile phase, and $20 \mathrm{mM}$ bis-Tris consisting of $5 \mathrm{mM}$ PTSA. We mixed $100 \mu \mathrm{M}$ ethylenediaminetetraacetic acid immediately before detection to enhance the sensitivity, and $1 \mathrm{mM}$ pimelic acid was used as an internal standard.

We performed HS/GC-MS via a GC-MS QP2010 Ultra instrument (Shimadzu) equipped with a DB-624 $(60 \mathrm{~m} \times 0.32 \mathrm{~mm} \times 1.8 \mu \mathrm{m})$ capillary column (Agilent Technologies, Santa Clara, CA, USA) and a HS-20 headspace sampler. We used helium as the carrier gas, and the flow rate was maintained at $75.9 \mathrm{~mL} \mathrm{~min}^{-1}$. The injection volume was $1 \mu \mathrm{L}$, with a split ratio of $1: 20$. We incubated the sample at $40^{\circ} \mathrm{C}$ for $15 \mathrm{~min}$. The oven temperature was initially $40^{\circ} \mathrm{C}$ for $3 \mathrm{~min}$, after which it was increased to $220^{\circ} \mathrm{C}$ at $60^{\circ} \mathrm{C} \mathrm{min}-1$, was maintained for $2 \mathrm{~min}$, and then was decreased to $40^{\circ} \mathrm{C}$ at $80^{\circ} \mathrm{C} \mathrm{min}-1$, at which point it was maintained for $1 \mathrm{~min}$. The total run time was $11 \mathrm{~min}$. The other settings were as follows: interface temperature, $250^{\circ} \mathrm{C}$; ion source temperature, $200^{\circ} \mathrm{C}$; and electron impact ionization, $70 \mathrm{eV}$. We calculated the 1,3-butadiene yield from the concentration of 1,3butadiene determined in the gas phase of the packed vial. For jar fermentation, we estimated the 1,3-butadiene yield from the concentration of 1,3-butadiene in the exhaust culture gases at each sampling point.

We performed GC-MS using a GC-MS QP2010 Ultra instrument (Shimadzu) equipped with a Pure-WAX $(32 \mathrm{~m} \times 0.25 \mathrm{~mm} \times 0.25 \mu \mathrm{m})$ column (GL Sciences, Japan). We used helium as the carrier gas, and the flow rate was maintained at 2.12 $\mathrm{mL} \mathrm{min}{ }^{-1}$. The injection volume was $1 \mu \mathrm{L}$, with a split ratio of $1: 5$. The oven temperature was initially $120^{\circ} \mathrm{C}$ for $1 \mathrm{~min}$, after which it was increased to $250^{\circ} \mathrm{C}$ at $30^{\circ} \mathrm{C} \mathrm{min}-1$ at which point it was maintained for $4 \mathrm{~min}$. The total run time was $10 \mathrm{~min}$. The other settings were as follows: interface temperature, $250^{\circ} \mathrm{C}$; ion source temperature, $200^{\circ} \mathrm{C}$; and electron impact ionization, $70 \mathrm{eV}$. We determined the PA concentration in the culture supernatant, which we separated from the culture broth by centrifugation at $15,000 \times g$ for $15 \mathrm{~min}$. We added $30 \mu \mathrm{L}$ of $1 \mathrm{M} \mathrm{HCl}$ to $300 \mu \mathrm{L}$ of the culture supernatant. Afterward, the mixture and $300 \mu \mathrm{L}$ of ethyl acetate were mixed together in a glass vial. After vortexing the mixture for $1 \mathrm{~min}$ and centrifuging it at $11,000 \times g$ for $1 \mathrm{~min}$, we analyzed the upper phase (Supplementary Fig. 12). We used $0.1 \mathrm{mM}$ hexanoic acid as an internal standard.

Reporting Summary. Further information on research design is available in the Nature Research Reporting Summary linked to this article.

\section{Data availability}

Data supporting the findings of this work are available within the paper and its Supplementary Information files. A reporting summary for this Article is available as a Supplementary Information file. The data that support the findings of this study are available from the corresponding author upon reasonable request. Source data are provided with this paper.

Received: 22 September 2020; Accepted: 18 March 2021; Published online: 13 April 2021

\section{References}

1. Woolston, B. M., Edgar, S. \& Stephanopoulos, G. Metabolic engineering: past and future. Annu. Rev. Chem. Biomol. Eng. 4, 259-288 (2013).

2. Mori, Y. \& Shirai, T. Designing artificial metabolic pathways, construction of target enzymes, and analysis of their function. Curr. Opin. Biotechnol. 54, 41-44 (2018)
3. Liao, J. C., Mi, L., Pontrelli, S. \& Luo, S. Fuelling the future: microbial engineering for the production of sustainable biofuels. Nat. Rev. Microbiol. 14, 288-304 (2016)

4. Noda, S. \& Kondo, A. Recent advances in microbial production of aromatic chemicals and derivatives. Trends Biotechnol. 35, 785-796 (2017).

5. Matsumoto, T., Tanaka, T. \& Kondo, A. Engineering metabolic pathways in Escherichia coli for constructing a "microbial chassis" for biochemical production. Bioresour. Technol. 245, 1362-1368 (2017).

6. Liu, Y. \& Nielsen, J. Recent trends in metabolic engineering of microbial chemical factories. Curr. Opin. Biotechnol. 60, 188-197 (2019).

7. Huccetogullari, D., Luo, Z. W. \& Lee, S. Y. Metabolic engineering of microorganisms for production of aromatic compounds. Microb. Cell Fact. 18, 41 (2019).

8. Cravens, A., Payne, J. \& Smolke, C. D. Synthetic biology strategies for microbial biosynthesis of plant natural products. Nat. Commun. 10, 2142 (2019).

9. Tan, Z. L. et al. In vivo continuous evolution of metabolic pathways for chemical production. Microb. Cell Factories 14, 18 (2019).

10. Lee, S. Y. et al. A comprehensive metabolic map for production of bio-based chemicals. Nat. Cat. 2, 18-33 (2019).

11. Sulzbach, M. \& Kunjapur, A. M. The pathway less traveled: engineering biosynthesis of nonstandard functional groups. Trends Biotechnol. 38, 532-545 (2020).

12. Qi, Y. et al. Synthesis of 1,3-butadiene and its 2-Substituted monomers for synthetic rubbers. Catalysts 9, 97 (2019).

13. Makshina, E. V. et al. Review of old chemistry and new catalytic advances in the on-purpose synthesis of butadiene. Chem. Soc. Rev. 43, 7917-7953 (2014).

14. Angelici, C., Weckhuysen, B. M. \& Bruijnincx, P. C. A. Chemocatalytic conversion of ethanol into butadiene and other bulk chemicals. ChemSusChem 6, 1595-1614 (2013).

15. Jang, Y. S. et al. Bio-based production of $\mathrm{C} 2$-C6 platform chemicals. Biotechnol. Bioeng. 109, 2437-2459 (2012).

16. Pomalaza, G., Capron, M., Ordomsky, V. \& Dumeignil, F. Recent breakthroughs in the conversion of ethanol to butadiene. Catalysts 6, 203 (2016).

17. Abdelrahman, O. A. et al. Biomass-derived butadiene by dehydra-decyclization of tetrahydrofuran. ACS Sustain. Chem. Eng. 5, 3732-3736 (2017).

18. Sun, D. et al. Production of 1,3-butadiene from biomass-derived C4 alcohols Fuel Process. Technol. 197, 106193 (2020).

19. Yang, J. et al. Metabolic engineering of Methylobacterium extorquens AM1 for the production of butadiene precursor. Microb. Cell Fact. 17, 194 (2018).

20. Khalil, I., Quintens, G., Junkers, T. \& Dusselier, M. Muconic acid isomers as platform chemicals and monomers in the biobased economy. Green. Chem. 22, 1517-1541 (2020)

21. Noda, S., Shirai, T., Oyama, S. \& Kondo, A. Metabolic design of a platform Escherichia coli strain producing various chorismate derivatives. Metab. Eng. 33, 119-129 (2016).

22. Noda, S., Shirai, T., Mori, Y., Oyama, S. \& Kondo, A. Engineering a synthetic pathway for maleate in Escherichia coli. Nat. Commun. 8, 1153 (2017).

23. Fujiwara, R., Noda, S., Tanaka, T. \& Kondo, A. Metabolic engineering of Escherichia coli for shikimate pathway derivative production from glucosexylose Co-substrate. Nat. Commun. 11, 1037 (2020).

24. Mukai, N., Masaki, K., Fujii, T., Kawamukai, M. \& Iefuji, H. PAD1 and FDC1 are essential for the decarboxylation of phenylacrylic acids in Saccharomyces cerevisiae. J. Biosci. Bioeng. 109, 564-569 (2010).

25. Payne, K. A. et al. New cofactor supports $\alpha, \beta$-unsaturated acid decarboxylation via 1,3-dipolar cycloaddition. Nature 522, 497-501 (2015).

26. Wang, P. H. et al. Biosynthesis and activity of prenylated FMN cofactors. Cell Chem. Biol. 25, 560-570.e6 (2018).

27. Bailey, S. S. et al. The role of conserved residues in Fdc decarboxylase in prenylated Flavin mononucleotide oxidative maturation, cofactor isomerization, and catalysis. J. Biol. Chem. 293, 2272-2287 (2018).

28. Marshall, S. A. et al. Oxidative maturation and structural characterization of prenylated FMN binding by UbiD, a decarboxylase involved in bacterial ubiquinone biosynthesis. J. Biol. Chem. 292, 4623-4637 (2017).

29. Payer, S. E. et al. Regioselectivepara-carboxylation of catechols with a prenylated Flavin dependent decarboxylase. Angew. Chem. Int. Ed. Engl. 56, 13893-13897 (2017)

30. Leys, D. Flavin metamorphosis: cofactor transformation through prenylation. Curr. Opin. Chem. Biol. 47, 117-125 (2018).

31. Payer, S. E., Faber, K. \& Glueck, S. M. Non-oxidative enzymatic (De) Carboxylation of (Hetero)Aromatics and acrylic acid derivatives. Adv. Synth. Catal. 361, 2402-2420 (2019).

32. Aleku, G. A. et al. Terminal alkenes from acrylic acid derivatives via nonoxidative enzymatic Decarboxylation by ferulic acid decarboxylases. ChemCatChem 10, 3736-3745 (2018)

33. Bailey, S. S. et al. Enzymatic control of cycloadduct conformation ensures reversible 1,3-dipolar cycloaddition in a prfmn-dependent decarboxylase. Nat. Chem. 11, 1049-1057 (2019). 
34. Balaikaite, A. et al. Ferulic acid decarboxylase controls oxidative maturation of the prenylated flavin mononucleotide cofactor. ACS Chem. Biol. 15, 2466-2475 (2020).

35. Saaret, A., Balaikaite, A. \& Leys, D. Biochemistry of prenylated-FMN enzymes. Flavin-dependent enzymes. Enzymes 47, 517-549 (2020).

36. Messiha, H. L., Payne, K. A. P., Scrutton, N. S. \& Leys, D. A biological route to conjugated alkenes: microbial production of hepta-1,3,5-triene. ACS Synth. Biol. 10, 228-235 (2021).

37. Kille, S., Zilly, F. E., Acevedo, J. P. \& Reetz, M. T. Regio- and stereoselectivity of P450-catalysed hydroxylation of steroids controlled by laboratory evolution. Nat. Chem. 3, 738-743 (2011).

38. Mutti, F. G., Knaus, T., Scrutton, N. S., Breuer, M. \& Turner, N. J. Conversion of alcohols to enantiopure amines through dual-enzyme hydrogen-borrowing cascades. Science 349, 1525-1529 (2015).

39. Pavlidis, I. V. et al. Identification of $(S)$-selective transaminases for the asymmetric synthesis of bulky chiral amines. Nat. Chem. 8, 1076-1082 (2016).

40. Yang, K. K., Wu, Z. \& Arnold, F. H. Machine-learning-guided directed evolution for protein engineering. Nat. Methods 16, 687-694 (2019).

41. Walther, T. et al. Construction of a synthetic metabolic pathway for biosynthesis of the non-natural methionine precursor 2,4-dihydroxybutyric acid. Nat. Commun. 8, 15828 (2017).

42. $\mathrm{Li}, \mathrm{R}$. et al. Computational redesign of enzymes for regio- and enantioselective hydroamination. Nat. Chem. Biol. 14, 664-670 (2018).

43. Lee, J. W., Niraula, N. P. \& Trinh, C. T. Harnessing a P450 fatty acid decarboxylase from Macrococcus caseolyticus for microbial biosynthesis of odd chain terminal alkenes. Metab. Eng. Commun. 7, e00076 (2018).

44. Kang, M. K. \& Nielsen, J. Biobased production of alkanes and alkenes through metabolic engineering of microorganisms. J. Ind. Microbiol. Biotechnol. 44, 613-622 (2017).

45. Nestl, B. M. et al. Structural and functional insights into asymmetric enzymatic dehydration of alkenols. Nat. Chem. Biol. 13, 275-281 (2017).

46. Chu, H. S. et al. Direct fermentation route for the production of acrylic acid. Metab. Eng. 32, 23-29 (2015).

47. Marchand, J. A. et al. Discovery of a pathway for terminal-alkyne amino acid biosynthesis. Nature 567, 420-424 (2019).

48. Ko, Y. S., Kim, J. W., Chae, T. U., Song, C. W. \& Lee, S. Y. A novel biosynthetic pathway for the production of acrylic acid through $\beta$-alanine route in Escherichia coli. ACS Synth. Biol. 9, 1150-1159 (2020).

49. St-Jacques, A. D., Eyahpaise, M. C. \& Chica, R. A. Computational design of Multisubstrate enzyme specificity. ACS Catal. 9, 5480-5485 (2019).

50. He, J., Huang, X., Xue, J. \& Zhu, Y. Computational redesign of penicillin acylase for cephradine synthesis with high kinetic selectivity. Green. Chem. 20, 5484-5490 (2018).

51. Voss, M. et al. Creation of ( $\mathrm{r}$ )-amine transaminase activity within an $\alpha$-amino acid transaminase scaffold. ACS Chem. Biol. 15, 416-424 (2020).

52. Tseliou, V., Knaus, T., Masman, M. F., Corrado, M. L. \& Mutti, F. G. Generation of amine dehydrogenases with increased catalytic performance and substrate scope from $\varepsilon$-deaminating L-lysine dehydrogenase. Nat. Commun. 10, 3717 (2019).

53. Vavricka, C. J. et al. Mechanism-based tuning of insect 3,4dihydroxyphenylacetaldehyde synthase for synthetic bioproduction of benzylisoquinoline alkaloids. Nat. Commun. 10, 2015 (2019).

54. Wang, H. et al. Improving the expression of recombinant proteins in E. coli BL21 (DE3) under acetate stress: an alkaline pH shift approach. PLoS ONE 9, e112777 (2014).

55. Ferguson, K. L., Arunrattanamook, N. \& Marsh, E. N. Mechanism of the novel prenylated Flavin-containing enzyme ferulic acid decarboxylase probed by isotope effects and linear free-energy relationships. Biochemistry 55, 2857-2863 (2016).

56. Mavrovounitis, M. L. Group contributions for estimating standard gibbs energies of formation of biochemical compounds in aqueous solution. Biotechnol. Bioeng. 36, 1070-1082 (1990).

57. Mavrovounitis, M. L. Estimation of standard Gibbs energy changes of biotransformations. J. Biol. Chem. 266, 14440-14445 (1991).
58. Stephanopoulos, G. N., Aristidou, A. A. \& Nielsen, J. H. Metabolic engineering principles and methodologies. (Acad. Press, 2008).

59. Antonovsky, N. et al. Sugar synthesis from $\mathrm{CO}_{2}$ in Escherichia coli. Cell 166, 115-125 (2016)

60. Gleizer, S. et al. Conversion of Escherichia coli to generate all biomass carbon from $\mathrm{CO}_{2}$. Cell 179, 1255-1263 (2019).

61. Martinez, K. A. et al. Cytoplasmic pH response to acid stress in individual cells of Escherichia coli and Bacillus subtilis observed by fluorescence ratio imaging microscopy. Appl. Environ. Microbiol. 78, 3706-3714 (2012).

62. Carraher, J. M., Pfennig, T., Rao, R. G., Shanks, B. H. \& Tessonnier, J.-P. cis, cis-Muconic acid isomerization and catalytic conversion to biobased cyclicC6-1,4-diacid monomers. Green. Chem. 19, 3042-3050 (2017).

\section{Acknowledgements}

This work was supported by the FY2017 Incentive Research Projects. We thank Yokohama Rubber Co., Ltd. (Kanagawa, Japan), and the ZEON Corporation (Tokyo, Japan) for financial support.

\section{Author contributions}

T.S. conceived and initiated the research. Y.M. designed and performed the experiments and wrote the paper. S.N. developed the strains, constructed the plasmids, and wrote the relevant methods. T.S. and A.K. supervised the research. All the authors have read and approved the manuscript.

\section{Competing interests}

RIKEN has filed a patent application related to 1,3-butadiene biosynthesis on behalf of Y.M. and T.S. The patent application number is JP 2019-532643. This study was funded by Yokohama Rubber Co., Ltd. (Kanagawa, Japan) and the ZEON Corporation (Tokyo, Japan). The other authors declare no competing interest.

\section{Additional information}

Supplementary information The online version contains supplementary material available at https://doi.org/10.1038/s41467-021-22504-6.

Correspondence and requests for materials should be addressed to T.S.

Peer review information Nature Communications thanks Junichi Kato, Min-Kyu Oh, and other, anonymous, reviewer(s) for their contributions to the peer review of this work. Peer review reports are available.

Reprints and permission information is available at http://www.nature.com/reprints

Publisher's note Springer Nature remains neutral with regard to jurisdictional claims in published maps and institutional affiliations.

pen Access This article is licensed under a Creative Commons Attribution 4.0 International License, which permits use, sharing, adaptation, distribution and reproduction in any medium or format, as long as you give appropriate credit to the original author(s) and the source, provide a link to the Creative Commons license, and indicate if changes were made. The images or other third party material in this article are included in the article's Creative Commons license, unless indicated otherwise in a credit line to the material. If material is not included in the article's Creative Commons license and your intended use is not permitted by statutory regulation or exceeds the permitted use, you will need to obtain permission directly from the copyright holder. To view a copy of this license, visit http://creativecommons.org/ licenses/by/4.0/

(C) The Author(s) 2021 